\title{
VECTOR VALUED MEAN-PERIODIC FUNCTIONS ON GROUPS
}

\author{
P. DEVARAJ and INDER K. RANA
}

(Received 12 March 2000; revised 20 March 2001)

\author{
Communicated by A. H. Dooley
}

\begin{abstract}
Let $G$ be a locally compact Hausdorff abelian group and $X$ be a complex Banach space. Let $C(G, X)$ denote the space of all continuous functions $f: G \rightarrow X$, with the topology of uniform convergence on compact sets. Let $X^{\prime}$ denote the dual of $X$ with the weak* topology. Let $M_{c}\left(G, X^{\prime}\right)$ denote the space of all $X^{\prime}$-valued compactly supported regular measures of finite variation on $G$. For a function $f \in C(G, X)$ and $\mu \in M_{c}\left(G, X^{\prime}\right)$, we define the notion of convolution $f \star \mu$. A function $f \in C(G, X)$ is called mean-periodic if there exists a non-trivial measure $\mu \in M_{c}\left(G, X^{\prime}\right)$ such that $f \star \mu=0$. For $\mu \in M_{c}\left(G, X^{\prime}\right)$, let $M P(\mu)=\{f \in C(G, X): f \star \mu=0\}$ and let $M P(G, X)=\bigcup_{\mu} M P(\mu)$. In this paper we analyse the following questions: Is $M P(G, X) \neq \emptyset$ ? Is $M P(G, X) \neq C(G, X)$ ? Is $M P(G, X)$ dense in $C(G, X)$ ? Is $M P(\mu)$ generated by 'exponential monomials' in it? We answer these questions for the groups $G=\mathbb{R}$, the real line, and $G=\mathbb{T}$, the circle group. Problems of spectral analysis and spectral synthesis for $C(\mathbb{R}, X)$ and $C(\mathbb{T}, X)$ are also analysed.
\end{abstract}

2000 Mathematics subject classification: primary 43A45; secondary 42A75.

Keywords and phrases: Convolution of vector valued functions, spectrum, vector valued mean-periodic functions, spectral synthesis.

\section{Introduction}

The notion of mean-periodic functions was introduced in 1935 by Delsarte [5]. It is well known that every solution of a constant coefficient homogeneous ordinary differential equation is a finite linear combination of solutions of the type $t^{k} e^{i \lambda t}$, where $\lambda \in \mathbb{C}$, and $k \in \mathbb{Z}_{+}$. Delsarte was interested in knowing whether this result is still true for convolution equation of the following type

$$
\int_{\mathbb{R}} f(s-t) k(t) d t=0, \quad \forall s \in \mathbb{R},
$$

The first author was supported by NBHM, Department of Atomic Energy, India.

(C) 2002 Australian Mathematical Society 1446-8107/2000 \$A2.00+0.00 
where $k$ is a continuous function which is zero out side some interval. For $\tau>0$, periodic continuous functions of period $\tau$ are solutions of the convolution equation

$$
\frac{1}{\tau} \int_{s-\tau / 2}^{s+\tau / 2} f(t) d t=0, \quad \forall s \in \mathbb{R} .
$$

For this reason Delsarte called the continuous functions which are solutions of equation (1) as mean-periodic. In [35], Schwartz observed that the mean-periodicity of a continuous function does not depend upon the function $k$, and he extended Delsarte's definition as follows:

Definition 1.1. A continuous function $f: \mathbb{R} \rightarrow \mathbb{C}$ is said to be mean-periodic if there exists a non-trivial regular measure $\mu$ of compact support and finite variation such that $(f \star \mu)(s)=\int_{\mathbb{R}} f(s-t) d \mu(t)=0, \forall s \in \mathbb{R}$.

Schwartz also gave an intrinsic characterization of mean-periodic functions. Let $C(\mathbb{R})$ denote the vector space of complex valued continuous functions on $\mathbb{R}$ with the topology of uniform convergence on compact sets (u.c.c.). Let $M_{c}(\mathbb{R})$ denote the space of all regular measures of compact support and finite variation on $\mathbb{R}$. For $f \in C(\mathbb{R})$, let $\tau(f)$ denote the closed translation invariant subspace of $C(\mathbb{R})$ generated by $f$. Schwartz in [35] showed that $f \in C(\mathbb{R})$ is mean-periodic if and only if $\tau(f) \neq C(\mathbb{R})$. Further, if $f \star \mu=0$ for some non-zero $\mu \in M_{c}(\mathbb{R})$, then $f$ is a limit of finite linear combination of exponential monomials $t^{k} e^{i \lambda t}$ which satisfy $t^{k} e^{i \lambda t} \star \mu=0$. More generally, convolution equation of the type

$$
f \star \mu=g,
$$

where $\mu \in M_{c}(\mathbb{R})$ and $g \in C(\mathbb{R})$ are given, can be analysed as in the case of ordinary differential equations. If $p$ is a particular solution of the equation (3), then every other solution is of the form $h+p$, where $h$ is a solution of the homogeneous equation $f \star \mu=0$. In general, equation (3) need not have any solution in $C(\mathbb{R})$. For instance, let $\mu$ be such that $d \mu(t)=\phi(t) d t$, where $\phi \in C_{c}^{\infty}(\mathbb{R})$, space of all infinitely differentiable functions on $\mathbb{R}$, and $g$ is a nowhere differentiable continuous function on $\mathbb{R}$. Some particular cases of (3) were analysed in [31,32]. In general, no necessary and sufficient conditions for the existence of solutions of equation (3) are known. A variant of the above problem is the following: Consider the following convolution equation

$$
f_{1} \star \mu_{1}=-f_{2} \star \mu_{2},
$$

where $\mu_{1}, \mu_{2} \in M_{c}(\mathbb{R})$ are given. Equation (4) can be written as a convolution equation for vector valued functions: let $\underline{f}=\left(f_{1}, f_{2}\right): \mathbb{R} \rightarrow \mathbb{C}^{2}$ and $\underline{\mu}=\left(\mu_{1}, \mu_{2}\right): \mathscr{B}_{\mathbb{R}} \rightarrow \mathbb{C}^{2}$. 
Then equation (4) is a homogeneous equation $\underline{f} \star \underline{\mu}=0$. This leads to consideration of vector valued mean-periodic functions, the main content of this paper. We consider such equations in a more general setting and analyse their solutions.

Let $G$ be a locally compact abelian group. Let $X$ be a complex Banach space and $X^{\prime}$ denote the weak ${ }^{*}$-dual of $X$. We denote by $\mathscr{B}_{G}$ the $\sigma$-algebra of Borel subsets of $G$. We recall some results on integration of functions $f: G \rightarrow X$ with respect to $X^{\prime}$-valued measures on $\mathscr{B}_{G}$, denoted by $M\left(G, X^{\prime}\right)$. For details one may refer Schmets [34]. Let $\mu \in M\left(G, X^{\prime}\right)$ and for every $x$, let $\mu_{x}$ denote the scalar measure on $\mathscr{B}_{G}$ defined by $\mu_{x}(E):=\langle x, \mu(E)\rangle$ for every $E \in \mathscr{B}_{G}$. The measure $\mu$ is said to be regular if $\mu_{x}$ is regular for every $x \in X$. For $E \in \mathscr{B}_{G}$, if $E=\bigcup_{i=1}^{n} E_{i}$ for some $E_{1}, E_{2}, \ldots, E_{n} \in \mathscr{B}_{G}$ such that $E_{i} \bigcap E_{j}=\emptyset$ for $i \neq j$, we call $\left\{E_{1}, E_{2}, \ldots, E_{n}\right\}$ a measurable partition of $E$. Let $\mathscr{P}(E)$ denote the set of all measurable partitions of E. Let

$$
V_{\mu}(E):=\sup \left\{\sum_{i=1}^{n}\left\|\mu\left(E_{i}\right)\right\|:\left\{E_{1}, E_{2}, \ldots, E_{n}\right\} \in \mathscr{P}(E)\right\} .
$$

The scalar measure $V_{\mu}$ is called the variation of $\mu$. We say $\mu$ has finite variation if $V_{\mu}(E)<+\infty$ for every $E \in \mathscr{B}_{G}$. Let $M\left(G, X^{\prime}\right)$ denote the set of all regular Borel measures $\mu$ on $G$ such that $\mu$ has finite variation. For $\mu \in M\left(G, X^{\prime}\right)$ the smallest closed set $S$ with $\mu(E)=0$ for every $E \in \mathscr{B}_{G}$ with $E \cap S=\emptyset$ is called the support of $\mu$. We write $S=\operatorname{supp}(\mu)$ if $S$ is the support of $\mu$. Let $M_{c}\left(G, X^{\prime}\right)$ denote the set of all $\mu \in M\left(G, X^{\prime}\right)$ such that support of $\mu$ is compact. Let $C(G, X)$ denote the space of all $X$-valued continuous functions on $G$ with the topology of uniform convergence on compact sets. Let $f \in C(G, X)$ and $\mu \in M_{c}\left(G, X^{\prime}\right)$ with $\operatorname{supp}(\mu) \subseteq K$, a compact set. Then there exists a sequence $\mathscr{P}_{k}(K):=\left\{B_{k_{1}}^{k}, B_{k_{2}}^{k}, \ldots, B_{k_{n}}^{k}\right\}$ of measurable partitions of $K$ with the following property : for arbitrary choice of $t_{i} \in B_{k_{i}}$, the sequence $\left\{\sum_{i=1}^{n}\left\langle f\left(t_{i}\right), \mu\left(B_{k_{i}}^{k}\right)\right\rangle\right\}_{k \geq 1}$ is convergent and is independent of the choice of $t_{i}^{\prime} s$. This limit is called the integral of $f$ with respect to $\mu$ and is denoted by $\int f d \mu$. For $f \in C(G, X)$ and $\mu \in M_{c}\left(G, X^{\prime}\right)$ the scalar valued function

$$
(f \star \mu)(g):=\int_{G} f(g-h) d \mu(h), \quad \forall g \in G
$$

is called the convolution of $f$ with $\mu$, that is, $(f \star \mu)(g)=\mu\left(f_{g}\right)=\left\langle\mu, f_{g}\right\rangle$, where $f_{g}(h)=f(g+h)$ and $\langle\mu, f\rangle=\mu(f)=\int_{G} f(-g) d \mu(g)$ is the duality pairing of $M_{c}\left(G, X^{\prime}\right)$ with $C(G, X)$.

Definition 1.2. We say $f \in C(G, X)$ is mean-periodic if there exists a non-trivial $\mu \in M_{c}\left(G, X^{\prime}\right)$ such that $(f \star \mu)(g)=\int_{G} f(g-h) d \mu(h)=0, \forall g \in G$.

The aim of this paper is to answer the following questions: let $M P(G, X)$ denote the space of all $X$-valued mean-periodic functions on $G$. 
- Is $M P(G, X) \neq \emptyset$ ? That is, when does there exist non-zero mean-periodic functions?

- Is $M P(G, X) \neq C(G, X)$ ? That is, do there exist continuous functions which are not mean-periodic?

- Is $M P(G, X)$ dense in $C(G, X)$ ? That is, how large is $M P(G, X)$ as a subspace of $C(G, X)$ ?

We answer these questions for the particular cases $G=\mathbb{R}$, in Section 2 and $G=\mathbb{T}$, circle group, in Section 3. Analysis of such questions for more general groups remain open.

The problem of analysing mean-periodic functions is also related to the problem of 'spectral analysis' and 'spectral synthesis'. In order to carry-out the analysis, we define next vector valued exponential monomials and exponential polynomials.

An additive function on a locally compact abelian group is a complex valued continuous function $a$ on $G$ such that $a\left(g_{1}+g_{2}\right)=a\left(g_{1}\right)+a\left(g_{2}\right)$ for all $g_{1}$ and $g_{2}$ in $G$. A polynomial on $G$ is a function of the form $p\left(a_{1}, a_{2}, \ldots, a_{m}\right)$, where $p$ is a polynomial in $m$ variables and $a_{1}, a_{2}, \ldots, a_{m}$ are additive functions on $G$. A monomial on $G$ is a function of the form $p\left(a_{1}, a_{2}, \ldots, a_{m}\right)$, where $p$ is a monomial in $m$ variables and $a_{1}, a_{2}, \ldots, a_{m}$ are additive functions on $G$. An exponential on $G$ is a non-zero continuous complex valued function $\omega$ such that $\omega\left(g_{1}+g_{2}\right)=\omega\left(g_{1}\right) \omega\left(g_{2}\right)$ for all $g_{1}$ and $g_{2}$ in $G$. An exponential monomial is a point-wise product of a monomial and an exponential. An exponential polynomial is a point-wise product of a polynomial and an exponential. The set of all exponentials is denoted by $\Omega$. Note that $\Omega \subset C(G)$.

We define exponential polynomials in $C(G, X)$ as follows:

DEFINITION 1.3. (i) We call $f \in C(G, X)$ an $X$-valued exponential if for every $g \in G, f(g)=\omega(g) x$ for some $\omega \in \Omega$ and $x \in X$.

(ii) We call $f \in C(G, X)$ an $X$-valued exponential monomial if for every $g \in G$, $f(g)=p(g) \omega(g) x$ for some $x \in X, p$ a monomial in $C(G)$ and $\omega$ an exponential in $C(G)$.

(iii) We call $f \in C(G, X)$ an $X$-valued exponential polynomial if for every $g \in G$, $f(g)=p(g) \omega(g) x$ for some $x \in X, p$ a polynomial in $C(G)$ and $\omega$ an exponential in $C(G)$.

EXAmPle 1. (1) Let $f \in C(\mathbb{R}, X)$. Then $f$ is an exponential if and only if for every $t \in \mathbb{R}, f(t)=e^{i \lambda t} x$ for some $\lambda \in \mathbb{C}$ and $x \in X . f$ is an exponential monomial if and only if for every $t \in \mathbb{R}, f(t)=t^{k} e^{i \lambda t} x$ for some $\lambda \in \mathbb{C}, k \in \mathbb{N}$ and $x \in X$. Finally, $f$ is an exponential polynomial if and only if for every $t \in \mathbb{R}, f(t)=p(t) e^{i \lambda t} x$ for some $\lambda \in \mathbb{C}$, polynomial $p(t)$ and $x \in X$. Thus the exponentials, exponential monomials and exponential polynomials are the scalar multiples of the ones defined by Schwartz [35]. 
(2) A function $f \in C(\mathbb{T}, X)$ is an exponential if and only if for every $t \in \mathbb{R}$, $f\left(e^{l t}\right)=e^{\text {tnt }} x$ for some non-negative integer $n$ and $x \in X$.

REMARK. We shall use the following convention: When $X=\mathbb{C}$ we choose the $x \in$ $X$ appearing in the exponential, exponential monomial and exponential polynomial to be the scalar constant 1 . The generality is not lost due to this choice, since if a closed translation invariant subspace contains an exponential or exponential monomial or exponential polynomial if and only if it contains their scalar multiples.

DEFINITION 1.4. Let $V$ be a closed translation invariant subspace of $C(G, X)$. We say

(i) spectral analysis holds for $V$ if $V$ contains an exponential;

(ii) spectral synthesis holds for $V$ if the linear span of the set of all exponential monomials in $V$ is dense in $V$;

(iii) if spectral analysis (synthesis) holds for every closed translation invariant subspace $V$ of $C(G, X)$, then we say that spectral analysis (synthesis) holds in $C(G, X)$.

DEFINITION 1.5. Let $V$ be a closed translation invariant subspace of $C(G, X)$ and $f \in C(G, X)$ be mean-periodic. Let $\tau(f)$ denote the closed translation invariant subspace of $C(G, X)$ generated by $f$.

(i) The spectrum of $V$ is defined to be the set of all exponential monomials in $V$ and is denoted by $\operatorname{spec}(V)$ or $\sigma(V)$.

(ii) The spectrum of $f$ is defined to be $\operatorname{spec}(\tau(f))$ and is denoted by $\operatorname{spec}(f)$ or $\sigma(f)$.

Some of the known results for spectral analysis and spectral synthesis for $G=\mathbb{R}^{n}$ are as follows: Let $E\left(\mathbb{R}^{n}\right)$ be the space of all infinitely differentiable functions on $\mathbb{R}^{n}$ in the topology of compact convergence of functions and their derivatives. Then its dual $E\left(\mathbb{R}^{n}\right)^{\prime}$ is the space of all compactly supported distributions on $\mathbb{R}^{n}$. Schwartz [35] proved the following theorem:

THEOREM 1.6 ([35]). In E( $\mathbb{R})$, every closed translation invariant subspace is the closure of finite linear combinations of the exponential monomials in it.

As a consequence of this theorem, the linear span of exponential monomials in every closed translation invariant subspace $V$ of $C(\mathbb{R})$ is dense in $V$. That is, spectral analysis and spectral synthesis hold in $C(\mathbb{R})$. Using this Schwartz [35] described mean-periodic functions on $\mathbb{R}$.

Let $V$ be the closed translation invariant subspace of $E\left(\mathbb{R}^{n}\right)$ generated by the solutions of the homogeneous constant coefficient partial differential equation $p(D) f=0$. Malgrange [28] proved that spectral synthesis holds for $V$. 
In 1975 Gurevich [17] proved that Theorem 1.6 cannot be extended for $\mathbb{R}^{n}, n>1$. Though Theorem 1.6 fails for $\mathbb{R}^{n}, n>1$, spectral analysis and spectral synthesis hold in $C(G)$ for certain groups, for example, for $G=\mathbb{Z}^{n}$ (see [26]) and for discrete abelian groups (see $[12,13])$. Consider the following example from [15].

EXAMPle $2([10,15])$. Define $f_{1}, f_{2}: \mathbb{R}^{2} \rightarrow \mathbb{C}$ by

$$
f_{1}\left(x_{1}, x_{2}\right):=1 \quad \text { and } \quad f_{2}\left(x_{1}, x_{2}\right):=x_{1}+x_{2}, \quad \forall\left(x_{1}, x_{2}\right) \in \mathbb{R}^{2} .
$$

Let $V$ be the closed translation invariant subspace of $C\left(\mathbb{R}^{2}\right)$ generated by $f_{1}$ and $f_{2}$. Then the spectrum of $V$ is $\left\{f_{1}\right\}$. But the closed linear span of the spectrum of $V$ is a proper subspace of $V$. Thus spectral synthesis fails in $C\left(\mathbb{R}^{2}\right)$ and spectral synthesis fails for $V$ even if $V$ is finite dimensional.

However, for certain closed translation invariant subspaces $V \subset C\left(\mathbb{R}^{2}\right)$ the linear span of all exponential polynomials in $V$ is dense in $V$. These subspaces are described in the following three theorems.

THEOREM 1.7 ([4]). Let $V$ be a closed translation and rotation invariant subspace of $C\left(\mathbb{R}^{2}\right)$. Then the linear span of exponential polynomials in $V$ is dense in $V$.

THEOREM 1.8 ([16]). Let $\mu \in M_{c}\left(\mathbb{R}^{n}\right)$. Then the linear span of exponential polynomials in $\tau_{\mu}:=\left\{f \in C\left(\mathbb{R}^{n}\right): f \star \mu=0\right\}$ is dense in $\tau_{\mu}$.

THEOREM 1.9 ([14]). Let $V$ be a finite dimensional translation invariant subspace of $C\left(\mathbb{R}^{n}\right)$. Then every element of $V$ is a finite linear combination of exponential polynomials.

The following question is raised in [15] and the answer is not known: Let $V$ be closed translation invariant subspace of $C\left(\mathbb{R}^{2}\right)$.

- Does there exist an exponential in $V$ ?

In Section 4, we answer this question affirmatively when $V$ is either finite dimensional or rotation invariant or $V=\tau_{\mu}:=\left\{f \in C\left(\mathbb{R}^{2}\right): f \star \mu=0\right\}$ for some $\mu \in M_{c}\left(\mathbb{R}^{2}\right)$.

Let $V$ be a closed translation invariant subspace of $C(G, X)$. Then the problems of spectral analysis and synthesis are the following:

- Is every exponential monomial in $C(G, X)$ mean-periodic?

- Are exponential monomials dense in $C(G, X)$ ?

- When does there exist an exponential monomial in $V$ ?

- When is the linear span of exponential monomials in $V$ dense $V$ ?

- Does there exist an exponential monomial solution for the convolution equation $f \star \mu=0$ for a given $\mu \in M_{c}\left(G, X^{\prime}\right)$ ?

We analyse these problems for $G=\mathbb{R}$ in Section 2 and $G=\mathbb{T}$ in Section 3 . 


\section{Mean-periodic functions on $G=\mathbb{R}$}

For $G=\mathbb{R}$ and $X=\mathbb{C}$, it is known (see Schwartz [35]) that $f \in C(\mathbb{R}, \mathbb{C})$ is meanperiodic if and only if $\tau(f)$, the closed translation invariant subspace of $C(\mathbb{R}, \mathbb{C})$ is proper. We first extend this result to $X$, arbitrary Banach space.

THEOREM 2.1. The following are equivalent:

(i) $f$ is mean-periodic;

(ii) $\tau(f) \neq C(\mathbb{R}, X)$.

ProOF. We use the fact that $C(\mathbb{R}, X)$ is a locally convex space and its dual is $M_{c}\left(\mathbb{R}, X^{\prime}\right)$. To show that (i) implies (ii): let $\mu \in M_{c}\left(\mathbb{R}, X^{\prime}\right)$ be non-trivial such that $f \star \mu=0$. Then $\mu(g)=0$ for every $g \in \tau(f)$. Hence $\tau(f) \neq C(\mathbb{R}, X)$, for otherwise $\mu(g)=0$ for every $g \in C(\mathbb{R}, X)$, which is not possible, since $\mu$ is non-trivial. The implication (ii) implies (i) follows from the Hahn-Banach theorem for locally convex spaces and the fact that $\tau(f)$ is a proper closed translation invariant subspace of $C(\mathbb{R}, X)$.

We show next that there exist nontrivial $X$-valued mean-periodic functions on $\mathbb{R}$.

Proposition 2.2. $M P(\mathbb{R}, X) \neq \emptyset$.

Proof. Let $0 \neq x \in X$ and $0 \neq x^{\prime} \in X^{\prime}$. Choose $g \in M P(\mathbb{R})$, scalar valued function mean-periodic with respect to some $\mu \in M_{c}(\mathbb{R})$. Define $v: \mathscr{B}_{\mathbb{R}} \rightarrow X^{\prime}$ by $\nu(E):=\mu(E) x^{\prime}$ and define $f: \mathbb{R} \rightarrow \mathbb{C}$ by $f(t):=g(t) x$. Then $\mu$ is a $X^{\prime}$-valued measure and $f$ is a continuous $X$-valued function with $f \star v=(g \star \mu)\left\langle x, x^{\prime}\right\rangle=0$. Thus $f$ is mean-periodic with respect to $v$.

We prove next that existence of functions which are not mean-periodic is related to the $X$ being separable.

THEOREM 2.3. $M P(\mathbb{R}, X)$ is a proper subset of $C(\mathbb{R}, X)$ if and only if $X$ is separable.

Proof. Suppose that $X$ is a non-separable complex Banach space and $f \in C(\mathbb{R}, X)$. Since $f$ continuous, $f(\mathbb{R})$ is separable and hence $\overline{[f(\mathbb{R})]}$ is separable. Since, for every $g \in \tau(f), g(\mathbb{R}) \subseteq \overline{[f(\mathbb{R})]}, \tau(f) \neq C(\mathbb{R}, X)$. Hence $f$ is mean-periodic.

Conversely, suppose that $X$ is separable. We show that $M P(\mathbb{R}, X) \neq C(\mathbb{R}, X)$. For every $n \in \mathbb{N}$, let

$$
f_{n}(t):=\sum_{j=1}^{\infty} a_{n j} e^{i \lambda_{n j} t}, \quad t \in \mathbb{R},
$$


where $\lambda_{n j}$ and $a_{n j}$ satisfy the following conditions:

(i) $0 \neq a_{n j} \in \mathbb{C}$.

(ii) $\lambda_{n j} \in[\alpha, \beta]$ for some $\alpha<\beta$.

(iii) $\left\{\lambda_{n j}: j \in \mathbb{N}\right\} \cap\left\{\lambda_{m j}: j \in \mathbb{N}\right\}=\emptyset$ for $m \neq n$ and for every $n,\left\{\lambda_{n j}\right\}_{j=1}^{\infty}$ has a limit $\lambda_{n} \in \mathbb{R}$.

(iv) The convergence in (5) is uniform on compact sets with each $f_{n}$ bounded by 1 .

(v) $\sum_{n=1}^{\infty} \sum_{j=1}^{\infty}\left|a_{n j}\right|<\infty$.

Let $\left\{x_{1}, x_{2}, \ldots\right\}$ be a dense subset of $X$. Define $f: \mathbb{R} \rightarrow X$ by

$$
f(t):=\sum_{n=1}^{\infty} \frac{1}{2^{n}\left(1+\left\|x_{n}\right\|\right)} f_{n}(t) x_{n}, \quad t \in \mathbb{R} .
$$

We show that $f$ is not mean-periodic. Since $\left\{e^{i \lambda_{n j} t}\right\}_{n, j=1}^{\infty}$ is an equicontinuous family, $\left\{f_{n}\right\}_{n=1}^{\infty}$ is an equicontinuous family. Therefore, for $\mu \in M_{c}\left(\mathbb{R}, X^{\prime}\right)$,

$$
f \star \mu=\sum_{n=1}^{\infty} \frac{1}{2^{n}\left(1+\left\|x_{n}\right\|\right)}\left(f_{n} x_{n}\right) \star \mu=\sum_{n=1}^{\infty} \frac{1}{2^{n}\left(1+\left\|x_{n}\right\|\right)} f_{n} \star \mu_{x_{n}} .
$$

Thus $f \star \mu=0$ if and only if

$$
\sum_{n=1}^{\infty} \frac{1}{2^{n}\left(1+\left\|x_{n}\right\|\right)}\left(f_{n} \star \mu_{x_{n}}\right)(t)=0, \quad \forall t \in \mathbb{R},
$$

that is, for every $t \in \mathbb{R}$,

$$
\sum_{n=1}^{\infty} \frac{1}{2^{n}\left(1+\left\|x_{n}\right\|\right)} \sum_{j=1}^{\infty} a_{n j} \hat{\mu}_{x_{n}}\left(\lambda_{n j}\right) e^{i \lambda_{n j} t}=0
$$

Let $S_{p q}(t)=\sum_{n=1}^{p} \sum_{j=1}^{q} e^{i \lambda_{n j} t} a_{n j} \hat{\mu}_{x_{n}}\left(\lambda_{n j}\right) / 2^{n}\left(1+\left\|x_{n}\right\|\right)$. Notice that $S_{p q}$ is almost periodic and its Fourier coefficients $a\left(S_{p q} ; \lambda\right)$ satisfy the following:

$$
a\left(S_{p q} ; \lambda\right)= \begin{cases}\frac{a_{n j} \hat{\mu}_{x_{n}}\left(\lambda_{n j}\right)}{2^{n}\left(1+\left\|x_{n}\right\|\right)} & \text { if } \lambda=\lambda_{n j}, 1 \leq n \leq p, 1 \leq j \leq q \\ 0 & \text { otherwise. }\end{cases}
$$

Since the convergence in (6) is uniform, the convergence in (7) also is uniform. Therefore $S_{p q}$ converges to 0 uniformly as $p, q \rightarrow \infty$. Further, the Fourier coefficients $a\left(S_{p q} ; \lambda\right)$ converges to 0 as $p, q \rightarrow \infty$ ([27]). In view of (8), $a\left(S_{p q} ; \lambda\right)=0$ for every $\lambda$. Moreover, $\hat{\mu}_{x_{n}}\left(\lambda_{n j}\right)=0$ for every $n$ and $j$. Since $\left\{\lambda_{n j}\right\}_{j=1}^{\infty}$ has limit point, this implies $\mu_{x_{n}}=0$ for all $n$. Therefore, $\mu=0$. Hence $f$ is not mean-periodic.

Let $f \in C(\mathbb{R}, X)$ and let $x^{\prime} \in X^{\prime}$. Then $x^{\prime} \circ f \in C(\mathbb{R})$. It is natural to ask the following question: Is $x^{\prime} \circ f$ mean-periodic for every $x^{\prime} \neq 0$ if $f$ is mean-periodic? We analyse this in the following theorem. 
THEOREM 2.4. For $f \in C(\mathbb{R}, X)$ and $x^{\prime}, y^{\prime} \in X^{\prime}$ with $x^{\prime} \neq y^{\prime}$ the following hold:

(i) If $x^{\prime} \circ f$ is mean-periodic, then $f$ is mean periodic.

(ii) If $x^{\prime} \circ f=y^{\prime} \circ f$, then $f$ is mean-periodic.

(iii) If $X=\mathbb{C}^{n}, n>1$, then $f$ is a finite sum of mean-periodic functions.

(iv) There exists $f \in M P\left(\mathbb{R}, \mathbb{C}^{n}\right)$ such that $x^{\prime} \circ f$ is not mean-periodic for any $x^{\prime} \in X^{\prime}, x^{\prime} \neq 0$.

Proof. (i) By Theorem 2.1, it suffices to show that $\tau(f) \neq C(\mathbb{R})$. For this, let $g \in C(\mathbb{R}), g \neq 0$ be such that $g \notin \tau\left(x^{\prime} \circ f\right)$. Choose $v \in X$ such that $\left\langle x^{\prime}, v\right\rangle \neq 0$ and define $h: \mathbb{R} \rightarrow X$ by $h(t)=g(t) v /\left\langle x^{\prime}, v\right\rangle$. Then $h$ is continuous and $\left(x^{\prime} \circ h\right)(t)=g(t)$. We show that $h$ is not in $\tau(f)$. If possible let, $h \in \tau(f)$. Then there exists $\sum c_{i} f_{t_{i}} \rightarrow h$, which implies $x^{\prime}\left(\sum c_{i} f_{t_{i}}\right) \rightarrow x^{\prime} \circ h=g$, a contradiction.

(ii) Choose $g \in C(\mathbb{R}, X)$ such that $x^{\prime}(g) \neq y^{\prime}(g)$. We show that $g \notin \tau(f)$. If possible, let $g \in \tau(f)$. Since $\sum c_{i} f_{t_{i}} \rightarrow g \Rightarrow x^{\prime}\left(\sum c_{i} f_{t_{i}}\right) \rightarrow x^{\prime}(g)$ and $y^{\prime}\left(\sum c_{i} f_{t_{i}}\right) \rightarrow y^{\prime}(g)$, and also since $x^{\prime}(f)=y^{\prime}(f), x^{\prime}\left(\sum c_{i} f_{t_{i}}\right)=y^{\prime}\left(\sum c_{i} f_{t_{i}}\right)$. This implies $x^{\prime}(g)=y^{\prime}(g)$, a contradiction.

(iii) Let $f=\left(f_{1}, f_{2}, \ldots, f_{n}\right)$. Obviously $\left(0, \ldots, 0, f_{i}, 0, \ldots, 0\right)$ is mean-periodic for every $i$ with respect to $\mu=\left(\mu_{1}, \ldots, \mu_{n}\right)$ where $0 \neq \mu_{j} \in M_{c}(\mathbb{R})$ are arbitrary and for $j=i, \mu_{j}=0$. Hence $f$ is a finite sum of mean-periodic functions.

(iv) Choose a non zero, compactly supported complex valued continuous function $g$. Let $f=(g, g, \ldots, g)$. Then $f$ is a $\mathbb{C}^{n}$-valued continuous function on $\mathbb{R}$. Clearly $f$ is mean-periodic with respect to $\mu=\left(v_{1},-v_{1}, 0, \ldots, 0\right)$, where $0 \neq v_{1} \in M_{c}(\mathbb{R})$ is arbitrary but $x^{\prime} \circ f$ is not mean periodic for any $0 \neq x^{\prime} \in X^{\prime}$.

REMARK. When $X=\mathbb{C}, M P(\mathbb{R}, X)$ is a subspace of $C(\mathbb{R}, X)$. It follows from Theorem 2.4 (iii) that sum of mean-periodic functions in $C(\mathbb{R}, X)$ need not be meanperiodic and hence $M P(\mathbb{R}, X)$ in general need not be a vector subspace of $C(\mathbb{R}, X)$. Moreover, the same argument works for separable complex Hilbert spaces.

THEOREM 2.5. $M P(\mathbb{R}, X)$ is dense in $C(\mathbb{R}, X)$.

Proof. Case (i): $X=\mathbb{C}$. It suffices to show that the annihilator of $M P(\mathbb{R})$ is $\{0\}$. Let $\mu \in M_{c}(\mathbb{R})$ be such that $\mu(M P(\mathbb{R}))=\{0\}$. In particular $\mu\left(e^{i \lambda t}\right)=\hat{\mu}(\lambda)=0$ for every $\lambda \in \mathbb{C}$. Hence $\mu=0$.

Case (ii): Let $X$ be finite dimensional, $X=\mathbb{C}^{n}$. Consider $C(\mathbb{R}) \times C(\mathbb{R}) \times$ $\cdots \times C(\mathbb{R})$. This is a finite product of locally convex spaces. Hence it is a locally convex space in the product topology. It is easy to see that $C(\mathbb{R}, X)$ is isomorphic to $C(\mathbb{R}) \times \cdots \times C(\mathbb{R})$ as locally convex spaces. Also $M P(\mathbb{R}) \times M P(\mathbb{R}) \times \cdots \times M P(\mathbb{R}) \subseteq$ $M P(\mathbb{R}, X)$ and $M P(\mathbb{R})$ is dense in $C(\mathbb{R})$. Thus it follows that $M P(\mathbb{R}, X)$ is dense in $C(\mathbb{R}, X)$. 
Case (iii): $X$ is not finite dimensional. Consider the $\operatorname{set} \operatorname{Exp}(\mathbb{R}, X)=\left\{e^{i \lambda t} x: \lambda \in\right.$ $\mathbb{C}, x \in X\}$. We show that the linear span of $\operatorname{Exp}(\mathbb{R}, X)$ is contained in $M P(\mathbb{R}, X)$ and it is dense in $C(\mathbb{R}, X)$. Let $f(t)=e^{i \lambda_{1} t} x_{1}, g(t)=e^{\iota \lambda_{2} t} x_{2} \in \operatorname{Exp}(\mathbb{R}, X)$ and $\alpha, \beta \in \mathbb{C}$. Choose $0 \neq x^{\prime} \in X^{\prime}$ such that $x^{\prime}\left(x_{1}\right)=x^{\prime}\left(x_{2}\right)=0$ and $\mu_{1}, \mu_{2} \in M_{c}(\mathbb{R})$ such that $e^{i \lambda_{1} t} \star \mu_{1}=0=e^{i \lambda_{2} t} \star \mu_{2}$. Define $\mu(E)=\left(\mu_{1} \star \mu_{2}\right)(E) x^{\prime}$, for every $E \in \mathscr{B}_{\mathbb{R}}$. Then $(\alpha f+\beta g) \star \mu=0$. To prove the denseness, let $\mu \in M_{c}\left(\mathbb{R}, X^{\prime}\right)$ be such that $\mu$ annihilates the linear span of $\operatorname{Exp}(\mathbb{R}, X)$. Then $\hat{\mu}_{x}(\lambda)=0, \forall \lambda \in \mathbb{C}, \forall x \in X$. It follows that $\mu=0$. This completes the proof.

We analyse next the problem of spectral analysis and spectral synthesis in $C(\mathbb{R}, X)$. Let $V$ be a closed translation invariant subspace of $C(\mathbb{R}, X)$. For $X=\mathbb{C}$, Schwartz [35] proved that $V$ contains exponential monomials and the linear span of exponential monomials in $V$ is dense in $V$. It is well known [17] that spectral synthesis fails for $\mathbb{R}^{n}, n>1$. Further, it holds for certain locally compact abelian groups, namely for $\mathbb{Z}^{n}$ due to Lefranc [26] and discrete groups due to Gilbert [16, 15] and Elliott $[12,13]$. However, nothing is known for vector valued functions. In this section, we extend Schwartz's result for finite dimensional closed translation invariant subspace of $C(\mathbb{R}, X), X$ an arbitrary Banach space. For this we need the following lemmas.

LEMMA 2.6. Let $v^{1}, v^{2}, \ldots, v^{n} \in X^{n}, v^{i}=\left(v_{1}^{i}, v_{2}^{i}, \ldots, v_{n}^{i}\right)$, be linearly independent. Then there exist $x_{1}^{\prime}, x_{2}^{\prime}, \ldots, x_{n}^{\prime} \in X^{\prime}$ which satisfy

$$
\begin{aligned}
& x_{1}^{\prime}\left(v_{1}^{1}\right)+x_{2}^{\prime}\left(v_{2}^{1}\right)+\cdots+x_{n}^{\prime}\left(v_{n}^{1}\right)=1, \\
& x_{1}^{\prime}\left(v_{1}^{2}\right)+x_{2}^{\prime}\left(v_{2}^{2}\right)+\cdots+x_{n}^{\prime}\left(v_{n}^{2}\right)=0, \\
& \cdots \cdots \cdots \cdots \cdots \cdots \cdots \cdots \cdots+\cdots \cdots+x_{n}^{\prime}\left(v_{n}^{n}\right)=0 . \\
& x_{1}^{\prime}\left(v_{1}^{n}\right)+x_{2}^{\prime}\left(v_{2}^{n}\right)+\cdots \cdots
\end{aligned}
$$

Proof. Let $Y$ be the linear span of $\left\{v^{2}, v^{3}, \ldots, v^{n}\right\}$. Then $Y$ being a finite dimensional subspace of $X^{n}$ is closed. Since $v^{1}, v^{2}, \ldots, v^{n}$ are linearly independent, $v^{1} \notin Y$. Thus by Hahn-Banach theorem, there exists $\Lambda \in\left(X^{n}\right)^{\prime}$ such that $\Lambda(Y)=\{0\}$ and $\Lambda\left(v^{1}\right)=1$. Clearly $\Lambda$ can be written as $\Lambda=\left(x_{1}^{\prime}, x_{2}^{\prime}, \ldots, x_{n}^{\prime}\right)$, where $x_{i}^{\prime} \in X^{\prime}$ satisfy $\Lambda\left(x_{1}, x_{2}, \ldots, x_{n}\right)=x_{1}^{\prime}\left(x_{1}\right)+x_{2}^{\prime}\left(x_{2}\right)+\cdots+x_{n}^{\prime}\left(x_{n}\right)$. Therefore,

$$
\begin{aligned}
x_{1}^{\prime}\left(v_{1}^{1}\right)+x_{2}^{\prime}\left(v_{2}^{1}\right)+\cdots+x_{n}^{\prime}\left(v_{n}^{1}\right) & =\Lambda\left(v_{1}^{1}, 0, \ldots, 0\right)+\cdots+\Lambda\left(0, \ldots, 0, v_{n}^{1}\right) \\
& =\Lambda\left(\left(v_{1}^{1}, v_{2}^{1}, \ldots, v_{n}^{1}\right)\right)=1 .
\end{aligned}
$$

For every $i, 2 \leq i \leq n$,

$$
\begin{aligned}
x_{1}^{\prime}\left(v_{1}^{i}\right)+x_{2}^{\prime}\left(v_{2}^{i}\right)+\cdots+x_{n}^{\prime}\left(v_{n}^{i}\right) & =\Lambda\left(v_{1}^{i}, 0, \ldots, 0\right)+\cdots+\Lambda\left(0, \ldots, 0, v_{n}^{i}\right) \\
& =\Lambda\left(\left(v_{1}^{i}, v_{2}^{i}, \ldots, v_{n}^{i}\right)\right)=0 .
\end{aligned}
$$

This completes the proof of the lemma. 
For sets $A$ and $B$, let $\mathscr{F}(A, B)$ denote the set of all functions from $A$ to $B$. For a set $E \subseteq V$, a vector space, let $L S(E)$ denote the linear span of $E$.

LEMMA 2.7. Let $S$ be any set containing at-least $n$ points and $V$ be a vector space over $\mathbb{C}$. Let $\left\{f_{1}, f_{2}, \ldots, f_{n}\right\} \subset \mathscr{F}(S, V)$. Then $\left\{f_{1}, f_{2}, \ldots, f_{n}\right\}$ is linearly independent in $\mathscr{F}(S, V)$ if and only if there exists $n$ distinct points $t_{1}, t_{2}, \ldots, t_{n} \in S$ such that $\left\{f_{1}, f_{2}, \ldots, f_{n}\right\}$ is linearly independent in $\mathscr{F}\left(\left\{t_{1}, t_{2}, \ldots, t_{n}\right\}, V\right)$.

PROOF. We prove the straight implication by induction. Suppose that $\left\{f_{1}, f_{2}, \ldots\right.$, $\left.f_{n}\right\}$ is a linearly independent set in $\mathscr{F}(S, V)$. As $\left\{f_{1}\right\}$ is linearly independent, there exists $t_{1} \in S$ such that $f_{1}\left(t_{1}\right) \neq 0$. Then $\left\{f_{1}\right\}$ is linearly independent on $\left\{t_{1}\right\}$. Thus the lemma is true when $n=1$. If $f_{1}\left(t_{1}\right)=\alpha f_{2}\left(t_{1}\right)$, for some nonzero $\alpha \in \mathbb{C}$, choose $t_{2} \in S$ such that $f_{1}\left(t_{2}\right) \neq \alpha f_{2}\left(t_{2}\right)$, which is possible, since $f_{1}, f_{2}, \ldots, f_{n}$ are linearly independent on $S$. Then it is easy to check that $\left\{f_{1}, f_{2}\right\}$ is linearly independent on $\left\{t_{1}, t_{2}\right\}$. If $f_{1}\left(t_{1}\right) \neq \alpha f_{2}\left(t_{1}\right)$ for any non zero scalar and $f_{2}\left(t_{1}\right) \neq 0$, then choose any $t_{2} \neq t_{1}$. It is easy to see that $\left\{f_{1}, f_{2}\right\}$ is linearly independent on $\left\{t_{1}, t_{2}\right\}$. If $f_{2}\left(t_{1}\right)=0$, then choose $t_{2}$ such that $f_{2}\left(t_{2}\right) \neq 0$. In this case also one can easily verify that $\left\{f_{1}, f_{2}\right\}$ is linearly independent on $\left\{t_{1}, t_{2}\right\}$. Assume that $\left\{f_{1}, f_{2}, \ldots, f_{n-1}\right\}$ is linearly independent on $\left\{t_{1}, t_{2}, \ldots, t_{n-1}\right\}$. If $\left\{f_{1}, f_{2}, \ldots, f_{n-1}, f_{n}\right\}$ is linearly independent on $\left\{t_{1}, t_{2}, \ldots, t_{n-1}\right\}$ then choose any $t_{n}$ which is different from $t_{1}, t_{2}, \ldots, t_{n-1}$. If $\left\{f_{1}, f_{2}, \ldots, f_{n-1}, f_{n}\right\}$ is linearly dependent, then there exist unique scalars $\alpha_{1}, \alpha_{2}, \ldots, \alpha_{n-1}$ such that $\alpha_{1} f_{1}+\alpha_{2} f_{2}+\ldots+\alpha_{n-1} f_{n-1}=f_{n}$ on $\left\{t_{1}, t_{2}, \ldots, t_{n-1}\right\}$. Since $\left\{f_{1}, f_{2}, \ldots, f_{n}\right\}$ is linearly independent on $S$, there exists $t_{n} \in S$ such that $\alpha_{1} f_{1}\left(t_{n}\right)+\alpha_{2} f_{2}\left(t_{n}\right)+$ $\cdots+\alpha_{n-1} f_{n-1}\left(t_{n}\right) \neq f_{n}\left(t_{n}\right)$. It follows from this that $\left\{f_{1}, f_{2}, \ldots, f_{n}\right\}$ is linearly independent on $\left\{t_{1}, t_{2}, \ldots, t_{n}\right\}$. This proves the required claim.

The converse is trivial.

Using these lemmas we prove that every finite dimensional translation invariant subspace $V$ of $C(\mathbb{R}, X)$ includes an exponential and every element in $V$ is a finite sum of exponential monomials.

THEOREM 2.8. Let $V$ be an $n$-dimensional translation invariant subspace of $C(\mathbb{R}, X)$. Then the following hold:

(i) There exist $\lambda_{1}, \lambda_{2}, \ldots, \lambda_{q} \in \mathbb{C}$ and $m_{1}, m_{2}, \ldots, m_{q} \in \mathbb{N}$ with $m_{1}+m_{2}+\cdots+$ $m_{q}=n$, and $w_{1}, w_{2}, \ldots, w_{q} \in X$, not all zero, such that $e^{i \lambda_{j} t} w_{j} \in V$, for $1 \leq j \leq q$.

(ii) There exist $\lambda_{1}, \lambda_{2}, \ldots, \lambda_{q} \in \mathbb{C}, m_{1}, m_{2}, \ldots, m_{q} \in \mathbb{N}$ with $m_{1}+m_{2}+\cdots+$ $m_{q}=n$ and $x_{1}, x_{2}, \ldots, x_{n} \in X$ such that every $f \in V$ is of the form $f=\sum_{l=1}^{n} g_{l} x_{l}$, where each $g_{l} \in L S\left\{t^{k} e^{l \lambda_{j} t}: 0 \leq k \leq m_{j}-1,1 \leq j \leq q\right\}$.

(iii) There exist $\lambda_{1}, \lambda_{2}, \ldots, \lambda_{q} \in \mathbb{C}$ and $m_{1}, m_{2}, \ldots, m_{q} \in \mathbb{N}$ with $m_{1}+m_{2}+\cdots+$ $m_{q}=n$ such that every $f \in V$ is of the form $f=\sum_{j=1}^{q} \sum_{k=0}^{m_{j}-1} \alpha_{k j} t^{k} e^{i \lambda_{j} t} y_{k j}$, where $\alpha_{k j} \in \mathbb{C}$ and $y_{k j} \in X$ for $0 \leq k \leq m_{j}-1,1 \leq j \leq q$. 
Proof. Fix a basis $\left\{f_{1}, f_{2}, \ldots, f_{n}\right\}$ of $V$. Since $V$ is translation invariant, $\left(f_{i}\right)_{s} \in V$ for every $s \in \mathbb{R}$. Therefore there exist unique scalars $\alpha_{i j} \in \mathbb{C}$ such that $\left(f_{i}\right)_{s}=$ $\sum_{j=1}^{n} \alpha_{i j}(s) f_{j}$. Let $f$ denote the $n \times 1$ matrix $f=\left[f_{1}, f_{2}, \ldots, f_{n}\right]^{t}$ and $A(s)$ denote the $n \times n$ matrix $\left(\alpha_{i j}(s)\right)$. Then

$$
f_{s}=A(s)\left[f_{1}, f_{2}, \ldots, f_{n}\right]^{t}=A(s) f .
$$

Now

$$
\left(f_{s}-f\right) / s=((A(s)-A(0)) / s) f .
$$

Claim. $s \mapsto A(s)$ is continuous. We give two proofs of this claim.

PROOF 1. By the Lemma 2.7 there exist $n$ distinct points $\left\{t_{1}, t_{2}, \ldots, t_{n}\right\} \subset \mathbb{R}$ such that $\left\{f_{1}, f_{2}, \ldots, f_{n}\right\}$ is linearly independent on $\left\{t_{1}, t_{2}, \ldots, t_{n}\right\}$. In view of (9), $f\left(s+t_{j}\right)=A(s) f\left(t_{j}\right)$ for $j=1,2, \ldots, n$. That is $\left(f_{i}\left(s+t_{j}\right)\right)_{i, j=1}^{n}=A(s)\left(f_{i}\left(t_{j}\right)\right)_{i, j=1}^{n}$. Let $v^{i}=\left(f_{i}\left(t_{1}\right), f_{i}\left(t_{2}\right), \ldots, f_{i}\left(t_{n}\right)\right), 1 \leq i \leq n$. Then $\left\{v^{1}, v^{2}, \ldots, v^{n}\right\}$ is a linearly independent subset of $X^{n}$. By the Lemma 2.6 there exists $x_{i j}^{\prime} \in X^{\prime}$ such that

$$
\sum_{k=1}^{n}\left\langle f_{i}\left(t_{k}\right), x_{k j}^{\prime}\right\rangle=\delta_{i j}, \quad \text { where } \delta_{i j}= \begin{cases}1 & \text { if } i=j \\ 0 & \text { if } i \neq j\end{cases}
$$

Thus we have

$$
\left(f_{i}\left(s+t_{j}\right)\right)_{i, j=1}^{n}\left(x_{i j}^{\prime}\right)_{i, j=1}^{n}=A(s)\left(f_{i}\left(t_{j}\right)\right)_{i, j=1}^{n}\left(x_{i j}^{\prime}\right)_{i, j=1}^{n}=A(s)\left(\delta_{i j}\right)_{i, j=1}^{n}=A(s) .
$$

The entries of the matrix obtained by multiplying the matrices on the left side of the above equation are continuous. This shows that $s \mapsto A(s)$ is continuous from $\mathbb{R}$ to $B L\left(\mathbb{C}^{n}\right)$.

PROOF 2. For every $t \in \mathbb{R}$, define an operator $T_{t}: V \rightarrow V$ by

$$
\left(T_{t} f\right)(s):=f(t+s), \quad \forall f \in V, s \in \mathbb{R} .
$$

Then $T_{t} \in B L(V)$ and satisfies the following properties: For every $s, t \in \mathbb{R}$

(i) $T_{s} \circ T_{t}=T_{s+t}$;

(ii) $T_{0}=I$;

(iii) $T_{s} \circ T_{t}=T_{t} \circ T_{s}$.

Let $\left\{t_{1}, t_{2}, \ldots, t_{n}\right\}$ be as given by Lemma 2.7. Let $\left\{K_{n}\right\}_{n \geq 1}$ be compact subsets of $\mathbb{R}$ such that $\bigcup_{m=1}^{\infty} K_{m}=\mathbb{R}$ with $\left\{t_{1}, t_{2}, \ldots, t_{n}\right\} \subseteq K_{1} \subseteq K_{2} \subseteq \cdots$. To show the required claim we have to show that $t \mapsto T_{t}$ is continuous in $B L(V)$. We shall show first that $t \mapsto T_{t}$ is continuous point-wise. Let $s_{n} \rightarrow s$ as $n \rightarrow \infty$. Now $T_{s_{n}}(f)=f_{s_{n}}$ and $T_{s}(f)=f_{s}$, for every $f \in V$. Since $f$ is uniformly continuous on compact sets, $f_{s_{n}} \rightarrow f_{s}$ in $C(\mathbb{R}, X)$. Therefore $T_{s_{n}} \rightarrow T_{s}$ point-wise. To show that $T_{s_{n}} \rightarrow T_{s}$ in $B L(V)$, it is sufficient to show that for every $m,\left\|T_{s_{n}}-T_{s}\right\|_{K_{m}} \rightarrow 0$ as $n \rightarrow \infty$, where 
$\left\|T_{S_{n}}-T_{s}\right\|_{K_{m}}=\sup _{\|f\|_{K_{m} \leq 1}}\left\|T_{s_{n}}(f)-T_{s}(f)\right\|_{K_{m}}$. Let $\epsilon>0$. Since $\left\{f_{1}, f_{2}, \ldots, f_{n}\right\}$ is a basis of $V$, for every $f \in V$, there exist unique scalars $\alpha_{1}, \alpha_{2}, \ldots, \alpha_{n} \in \mathbb{C}$ such that $f=\alpha_{1} f_{1}+\alpha_{2} f_{2}+\cdots+\alpha_{n} f_{n}$. Also since $\left\{f_{1}, f_{2}, \ldots, f_{n}\right\}$ is linearly independent on $K_{m},\left\{\left(\alpha_{1}, \alpha_{2}, \ldots, \alpha_{n}\right) \in \mathbb{C}^{n}:\left\|\alpha_{1} f_{1}+\alpha_{2} f_{2}+\cdots+\alpha_{n} f_{n}\right\|_{K_{m}} \leq 1\right\}$ is bounded in $\mathbb{C}^{n}$, that is, there exists $M>0$ such that $\left\|\alpha_{1} f_{1}+\alpha_{2} f_{2}+\cdots+\alpha_{n} f_{n}\right\|_{K_{m}} \leq 1$ implies that $\left\|\left(\alpha_{1}, \alpha_{2}, \ldots, \alpha_{n}\right)\right\| \leq M$. Since $\left\{f_{1}, f_{2}, \ldots, f_{n}\right\}$ is equicontinuous, there exists a $\delta>0$, with $\delta<1$, such that whenever $t_{1}, t_{2} \in s+K_{m}+[0,1]$ with $\left|t_{1}-t_{2}\right|<\delta$, $\left\|f_{j}\left(t_{1}\right)-f_{j}\left(t_{2}\right)\right\|<\epsilon / M$, for every $j=1,2, \ldots, n$. Choose $N \in \mathbb{N}$ such that $\left|s_{n}-s\right|<\delta$, whenever $n \geq N$. Then for every $f \in V$ with $\|f\|_{K_{m}} \leq 1$, for every $t \in K_{m}$, and $n \geq N$, we have

$$
\begin{aligned}
\left\|f_{s_{n}}(t)-f_{s}(t)\right\| & =\left\|f\left(s_{n}+t\right)-f(s+t)\right\| \\
& =\left\|\left(\alpha_{1} f_{1}+\cdots+\alpha_{n} f_{n}\right)\left(s_{n}+t\right)-\left(\alpha_{1} f_{1}+\cdots+\alpha_{n} f_{n}\right)(s+t)\right\| \\
& \leq\left|\alpha_{1}\right|\left\|f_{1}\left(s_{n}+t\right)-f_{1}(s+t)\right\|+\cdots+\left|\alpha_{n}\right|\left\|f_{n}\left(s_{n}+t\right)-f_{n}(s+t)\right\| \\
& \leq \epsilon .
\end{aligned}
$$

Thus $\left\|T_{s_{n}}-T_{s}\right\|_{K_{m}} \rightarrow 0$ as $n \rightarrow \infty$ for every $m$ and hence $T_{s_{n}} \rightarrow T_{s}$ in $B L(V)$ as $n \rightarrow \infty$. This completes the second proof of the claim.

Thus $A(s)$ satisfies the following properties:

(i) $s \mapsto A(s)$ is continuous.

(ii) $A(0)=I$.

(iii) $A(s+t)=A(s) A(t)=A(t) A(s)$.

Therefore, $s \mapsto A(s)$ is differentiable (refer [18]) and

$$
A(s)=e^{s A^{\prime}(0)} .
$$

By virtue of equations (10) and (11),

$$
f^{\prime}=A^{\prime}(0) f \text {. }
$$

This equation can be solved ([21]) and the solution is given by

$$
f(t)=e^{t A^{\prime}(0)}\left[x_{1}, x_{2}, \ldots, x_{n}\right]^{t} .
$$

Let $\lambda_{1}, \lambda_{2}, \ldots, \lambda_{q} \in \mathbb{C}$ be the eigen values of $A^{\prime}(0)$ with multiplicities $m_{1}, m_{2}, \ldots$, $m_{q}$, respectively. Let the Jordan canonical form of $A^{\prime}(0)$ be given by

$$
B A^{\prime}(0) B^{-1}=\left[\begin{array}{lllll} 
& & & & \\
& & & & \\
& J_{2} & & \\
& & \ddots & \\
& & & J_{q}
\end{array}\right],
$$


where $J_{1}, \ldots, J_{q}$ are the Jordan blocks of $A^{\prime}(0), B$ is an invertible matrix. This gives

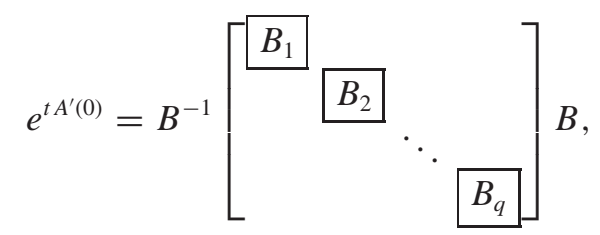

where each $B_{k}$ is an $m_{k} \times m_{k}$ matrix given by

$$
B_{k}=\left[\begin{array}{cccc}
e^{i \lambda_{k} t} & t e^{i \lambda_{k} t} & \cdots & e^{i \lambda_{k} t} m^{m_{k}-1} /\left(m_{k}-1\right) ! \\
0 & e^{i \lambda_{k} t} & \cdots & e^{i \lambda_{k} t} t^{m_{k}-2} /\left(m_{k}-2\right) ! \\
\vdots & \vdots & \ddots & \vdots \\
0 & 0 & \cdots & e^{i \lambda_{k} t}
\end{array}\right] .
$$

Thus $f(t)=C\left[x_{1}, x_{2}, \ldots, x_{n}\right]^{t}$, where $C=\left(c_{i j}\right)$ and each $c_{i j} \in L S\left\{t^{k} e^{i \lambda_{j} t}: 0 \leq\right.$ $\left.k \leq m_{j}-1,1 \leq j \leq q\right\}$, that is, for every $i, f_{i}(t)=\sum_{j=1}^{n} g_{i j}(t) x_{j}$, where $g_{i j} \in$ $L S\left\{t^{k} e^{i \lambda_{j} t}: 0 \leq k \leq m_{j}-1,1 \leq j \leq q\right\}$. Hence every element $h$ of $V$ is of the form $h(t)=\sum_{j=1}^{n} g_{j}(t) x_{j}$, where each $g_{j} \in L S\left\{t^{k} e^{i \lambda_{j} t}: 0 \leq k \leq m_{j}-1,1 \leq j \leq q\right\}$. This proves (ii).

(iii) By the discussion above, each $f_{i}$ can be expressed as follows:

$$
f_{i}=\sum_{j=1}^{q} \sum_{k=0}^{m_{j}-1} t^{k} e^{i \lambda_{j} t} \beta_{k j}^{i} x_{k j}^{i} .
$$

Every $h \in V$ is of the form

$$
\begin{aligned}
h & =\sum_{i=1}^{n} \alpha_{i} f_{i}=\sum_{i=1}^{n} \sum_{j=1}^{q} \sum_{k=0}^{m_{j}-1} t^{k} e^{i \lambda_{j} t} \alpha_{i} \beta_{k j}^{i} x_{k j}^{i} \\
& =\sum_{j=1}^{q} \sum_{k=0}^{m_{j}-1} t^{k} e^{i \lambda_{j} t}\left(\sum_{i=1}^{n} \alpha_{i} \beta_{k j}^{i} x_{k j}^{i}\right)=\sum_{j=1}^{q} \sum_{k=0}^{m_{j}-1} t^{k} e^{i \lambda_{j} t} y_{k j} .
\end{aligned}
$$

This proves (iii). For (i), $f_{i}=\sum_{j=1}^{q} \sum_{k=0}^{m_{j}-1} t^{k} e^{i \lambda_{j} t} y_{k j}^{i}$. For every $j$ choose largest $k$ such that $y_{k j}^{i} \neq 0$, let it be $k_{j}$. We will show that $e^{i \lambda_{j} t} y_{k_{j} j}^{i} \in V$. To prove this, let $\mu \in M_{c}\left(\mathbb{R}, X^{\prime}\right)$ be such that $\mu(V)=\{0\}$. Then $f \star \mu=0$ for every $f \in V$, since $V$ is translation invariant. Hence $f_{i} \star \mu=0$, for every $i$. As $f_{i} \star \mu$ is a finite sum of complex valued exponential monomials and $\hat{\mu}_{y_{k_{j} j}^{i}}\left(\lambda_{j}\right)$ is the coefficient of $e^{i \lambda_{j} t}, \hat{\mu}_{y_{k_{j}}^{i}}\left(\lambda_{j}\right)=0$. This implies that $e^{i \lambda t} y_{k_{j} j}^{i} \in V$.

COROllary 2.9. Let $f \in C(\mathbb{R}, X)$. Then $\tau(f)$ is finite dimensional if and only if $f$ is a finite linear combination of exponential monomials in $C(\mathbb{R}, X)$. 
Proof. Suppose that $\tau(f)$ is finite dimensional. Then it follows from the above theorem that $f$ is a finite linear combination of exponential monomials. Conversely, suppose $f$ is a finite linear combination of exponential monomials. Let $f=\sum_{j=1}^{q} \sum_{k=0}^{m_{j}-1} \alpha_{j k} t^{k} e^{l \lambda t} x_{j k}$. Then $\tau(f) \subseteq L S\left\{t^{k-l} e^{l \lambda_{j} t} x_{j k}: 0 \leq l \leq k, 0 \leq k \leq\right.$ $m_{j}-1,1 \leq j \leq q$ \}. Therefore $\tau(f)$ is finite dimensional.

REMARK. (i) Some authors (see [14, 25]) define exponential polynomials to be functions of the form $\sum_{j=1}^{m} f_{j}$, where $f_{j}$ are exponential polynomials defined as in Definition 1.3. With this definition, our result states that every finite dimensional translation invariant subspace $V$ of $C(\mathbb{R}, X)$ is generated by exponential polynomials in $V$.

(ii) Anselone and Korevaar [1] have proved that when $X=\mathbb{C}, V \subset C(\mathbb{R})$ is finite dimensional if and only if $V$ is the solution space of a homogeneous constant coefficient ordinary differential equation. This result is not true for arbitrary $X$ which can be seen by the following examples.

EXAMPLE 3. Let $X$ be a separable infinite dimensional complex Hilbert space. Let $\left\{e_{n}\right\}$ be a complete orthonormal basis. Consider the homogeneous ordinary differential equation with constant coefficient.

$$
a_{0} f+a_{1} f^{\prime}+\cdots+a_{n} f^{(n)}=0 .
$$

Let $\lambda_{1}, \lambda_{2}, \ldots, \lambda_{q}$ with multiplicities $m_{1}, m_{2}, \ldots, m_{q}$ be the roots of the characteristic polynomial $p(t)$. Then for every $n \in \mathbb{N}, 0 \leq k \leq m_{j}, 1 \leq j \leq q, t^{k} e^{i \lambda_{j} t} e_{n}$ is a solution of the differential equation (13). Thus the solution space is not finite dimensional.

EXAmple 4. Let $X$ be a complex Banach space. Fix $A \in B L(X)$. Consider the following differential equation $d u / d t=A u$. Then the solution space $\{u \in C(\mathbb{R}, X)$ : $d u / d t=A u\}=\left\{e^{t A} x: x \in X\right\}$ is a closed translation invariant subspace of $C(\mathbb{R}, X)$. Further, it is finite dimensional if and only if $X$ is finite dimensional.

Let $\mu \in M_{c}\left(\mathbb{R}, X^{\prime}\right)$. In the case when $X=\mathbb{C}$ it is known [35] that for a given $\mu$ the linear span of exponential monomial solutions of the convolution equation $f \star \mu=0$ is dense in the space of all solutions. We extend this for $X=\mathbb{C}^{n}$ as follows:

THEOREM 2.10. Let $f=\left(f_{1}, f_{2}, \ldots, f_{n}\right) \in C\left(\mathbb{R}, \mathbb{C}^{n}\right)$ satisfies the following:

(i) $f_{j}$ is mean-periodic, for every $1 \leq j \leq n$;

(ii) $\sigma\left(f_{j}\right) \cap \sigma\left(f_{k}\right)=\emptyset$ for $j \neq k$.

Then $\tau(f)$ contains exponential monomials and the linear span of exponential monomials in $\tau(f)$ is dense in $\tau(f)$. 
PROOF. Clearly $\tau(f) \subseteq \tau\left(f_{1}\right) \times \tau\left(f_{2}\right) \times \cdots \times \tau\left(f_{n}\right)$. We show that these two sets are equal. Let $g \in \tau\left(f_{1}\right) \cap \tau\left(f_{2}\right)$. Then $\tau(g) \subseteq \tau\left(f_{1}\right) \cap \tau\left(f_{2}\right)$ and hence by Schwartz's theorem, $\tau\left(f_{1}\right) \cap \tau\left(f_{2}\right)=(0)$. Thus $\tau\left(f_{i}\right) \cap \tau\left(f_{j}\right)=(0)$ for $i \neq j$. Let $\mu \in M_{c}\left(\mathbb{R}, X^{\prime}\right)$ be such that $\mu(\tau(f))=\{0\}$. Let $\mu=\left(\mu_{1}, \mu_{2}, \ldots, \mu_{n}\right)$. Since $\tau(f)$ is translation invariant, $f \star \mu=\sum_{j=1}^{n} f_{j} \star \mu_{j}=0$. Let $e^{i \lambda t}, t e^{i \lambda t}, \ldots, t^{m_{1}-1} e^{\imath \lambda t} \in \tau\left(f_{1}\right)$ and $t^{m_{1}} e^{i \lambda t} \notin \tau\left(f_{1}\right)$. By Hahn-Banach theorem there exists a measure $v_{1} \in M_{c}(\mathbb{R})$ such that $v_{1}\left(\tau\left(f_{l}\right)\right)=\{0\}$ for every $l \neq 1$ and $v_{1}\left(e^{i \lambda t}\right) \neq 0$. Therefore $f_{l} \star v_{1}=0$, for $l \neq 1$. Now $f_{1} \star \mu_{1} \star v_{1}=(f \star \mu) \star \mu_{1}=0$. Therefore $\left(\hat{\mu}_{1} \hat{v}_{1}\right)(\lambda)=\left(\hat{\mu}_{1} \hat{v}_{1}\right)^{\prime}(\lambda)=$ $\cdots=\left(\hat{\mu}_{1} \hat{v}_{1}\right)^{\left(m_{1}-1\right)}(\lambda)=0$. As $\hat{\mu}_{1}(\lambda) \hat{v}_{1}(\lambda)=0$ and $\hat{v}_{1}(\lambda) \neq 0, \hat{\mu}_{1}(\lambda)=0$. Also $\left(\hat{\mu}_{1} \hat{v}_{1}\right)^{\prime}(\lambda)=0$ implies $\hat{\mu}_{1}^{\prime}(\lambda)\left(\hat{v}_{1}\right)(\lambda)+\hat{\mu}_{1}(\lambda) \hat{v}_{1}^{\prime}(\lambda)=0$. This implies $\hat{\mu}_{1}^{\prime}(\lambda)=0$. Similarly we can show that $\hat{\mu}_{1}^{\prime \prime}(\lambda)=\cdots=\hat{\mu}_{1}^{\left(m_{1}-1\right)}(\lambda)=0$. Thus $\lambda$ is a zero of $\hat{\mu}_{1}$ with multiplicity at-least $m_{1}$. This shows that $f_{1} \star \mu_{1}=0$. Similarly, $f_{j} \star \mu_{j}=0$ for every $j$. Thus $\mu\left(\tau\left(f_{1}\right) \times \tau\left(f_{2}\right) \times \cdots \times \tau\left(f_{n}\right)\right)=0$. It follows that $\tau(f)=$ $\tau\left(f_{1}\right) \times \tau\left(f_{2}\right) \times \cdots \times \tau\left(f_{n}\right)$. This completes the proof.

Corollary 2.11. Let $X=\mathbb{C}^{n}$. Let $f=\left(f_{1}, f_{2}, \ldots, f_{n}\right) \in C(\mathbb{R}, X)$ and $\mu \in$ $M_{c}\left(\mathbb{R}, X^{\prime}\right)$. Suppose that each $f_{j}$ is mean-periodic and $\sigma\left(f_{j}\right) \cap \sigma\left(f_{k}\right)=\emptyset$ for $j \neq k$. If $f \star \mu=0$, then $f$ is a finite linear combination of exponential monomials solutions.

PROOF. Since spectral synthesis holds for $\mathbb{R}, L S\left(\sigma\left(f_{j}\right)\right)$ is dense in $\tau\left(f_{j}\right)$, for every $j$. It is easy to see that $\sigma\left(f_{1}\right) \times \sigma\left(f_{2}\right) \times \cdots \times \sigma\left(f_{n}\right) \subset L S(E)$, where $E=\left\{t^{k} e^{i \lambda t} x\right.$ : $\left.x \neq 0, t^{k} e^{i \lambda t} x \star \mu=0\right\}$. Thus $\overline{L S(E)}=\tau\left(f_{1}\right) \times \tau\left(f_{2}\right) \times \cdots \times \tau\left(f_{n}\right)$. The required result follows from the Theorem 2.10.

EXAMPLE 5. (1) When $G=\mathbb{R}$ and $X=\mathbb{C}$, the notion of mean-periodic functions was introduced by Delsarte in 1935 [5]. In [35] Schwartz gave an intrinsic characterization of mean-periodic functions: $f \in C(\mathbb{R}, \mathbb{C})$ is mean-periodic if and only if $\tau(f)$, the closed translation invariant subspace of $C(\mathbb{R}, \mathbb{C})$ is proper. Clearly, for every $\lambda \in \mathbb{C}, f_{\lambda}(t)=e^{i \lambda t}, t \in \mathbb{R}$, is mean-periodic, $f \star \mu=0$ for $\mu=\delta_{0}-e^{i \lambda} \delta_{1}$, where $\delta_{x}$ denote the Dirac measure on $\mathbb{R}$ at $x \in \mathbb{R}$. Schwartz [35] showed that if $f \in C(\mathbb{R}, \mathbb{C})$ is mean-periodic with $f \star \mu=0$, then $f$ is a limit of finite linear combinations of functions of the type $f_{\lambda}(t)=t^{k} e^{i \lambda t}$, such that $f_{\lambda} \star \mu=0$. In Laird [22] it is shown that if $f \in C(\mathbb{R}, \mathbb{C})$ is mean-periodic and $g$ is an exponential polynomial, that is, $g(t)=p(t) e^{i \lambda t}$, where $p(t)$ is a polynomial, then $f g$ is mean-periodic.

(2) Let $G$ be a compact abelian group. Then every character of $G$ is mean-periodic, as observed in Rana [33].

(3) For $X=\mathbb{C}$, mean-periodic functions on various locally compact groups have been analysed by various authors (see $[2,3,5,7,10,11,17,19,20,23,24,22,29,30,36$, 38, 37, 39]). 
In general setting, even when $G=\mathbb{R}$ and $X$ is an arbitrary Banach space, nothing seem to be known.

NOTE. The following questions still remain unanswered:

(1) Let $V$ be a closed translation invariant subspace of $C(\mathbb{R}, X)$. Does $V$ always include a monomial exponential? Is $V$ the closed linear span of the monomial exponentials in it?

(2) The problem of finding solutions for $f \star \mu=g$, for a given $\mu$ and $g$, seems to be much more difficult even for the case $G=\mathbb{R}$ and $X=\mathbb{C}$ : Some particular situations are analysed in [31] and [32]. Another particular case is given in the next theorem.

THEOREM 2.12. For a given $\mu \in M_{c}(\mathbb{R})$ and $g$ a finite sum of exponential polynomials in $C(\mathbb{R})$, there exists $f \in C(\mathbb{R})$ such that $f \star \mu=g$.

PROOF. First suppose that $g$ is an exponential polynomial. Let $g(t)=e^{\iota \lambda t} \sum_{k=0}^{n} a_{k} t^{k}$. Let $Z(\hat{\mu})=\{\lambda \in \mathbb{C}: \hat{\mu}(\lambda)=0\}$. We say

(i) $\lambda \in Z(\hat{\mu})$ is of multiplicity 0 if $\hat{\mu}(\lambda) \neq 0$.

(ii) $\lambda \in Z(\hat{\mu})$ of multiplicity $m \in \mathbb{N}$, if $\hat{\mu}(\lambda)=0, \hat{\mu}^{\prime}(\lambda)=0, \ldots, \hat{\mu}^{(m-1)}(\lambda)=0$ and $\hat{\mu}^{(m)}(\lambda) \neq 0$.

Let $m$ be the multiplicity of $\lambda \in Z(\hat{\mu})$. Define $f(t):=\sum_{k=0}^{n} b_{k} t^{m+k} e^{i \lambda t}$, where

$$
\begin{aligned}
& b_{n}=\frac{(\iota)^{m}}{\left(\begin{array}{c}
n+m \\
m
\end{array}\right) \hat{\mu}^{(m)}(\lambda)} a_{n}, \quad b_{n-1}=\left[a_{n-1}-b_{n} \frac{\left(\begin{array}{c}
m+n \\
m+1
\end{array}\right) \hat{\mu}^{(m+1)}(\lambda)}{(\iota)^{m+1}}\right] \frac{(\iota)^{m}}{\left(\begin{array}{c}
m+n-1 \\
m
\end{array}\right) \hat{\mu}^{(m)}(\lambda)}, \ldots, \\
& b_{0}=\left[a_{0}-b_{1} \frac{\left(\begin{array}{c}
m+1 \\
m+1
\end{array}\right) \hat{\mu}^{(m+1)}(\lambda)}{(\iota)^{m+1}}-b_{2} \frac{\left(\begin{array}{c}
m+2 \\
m+2
\end{array}\right) \hat{\mu}^{(m+2)}(\lambda)}{(\iota)^{m+2}}-\cdots\right. \\
& \left.-b_{n} \frac{\left(\begin{array}{c}
m+n \\
m+n
\end{array}\right) \hat{\mu}^{(m+n)}(\lambda)}{(\iota)^{m+n}}\right] \frac{(\iota)^{m}}{\left(\begin{array}{c}
m \\
m
\end{array}\right) \hat{\mu}^{(m)}(\lambda)} .
\end{aligned}
$$

A simple computation of $f \star \mu$ gives $f \star \mu=g$. In the general case, suppose that $g=\sum_{j=1}^{p} g_{j}$, where $g_{j}(t)=p_{j}(t) e^{i \lambda_{j} t}$, for every $j$ and $\lambda_{k} \neq \lambda_{j}$ for $k \neq j$. Let $f_{j}$ be the exponential polynomial function corresponding to $g_{j}$ obtained as in the first case, that is, $f_{j} \star \mu=g_{j}$. Then $f=\sum_{j=1}^{p} f_{j}$ is a solution of the given convolution equation.

\section{Mean-periodic functions on $G=\mathbb{T}$}

We shall consider integrals of $X$-valued functions with respect to scalar measures in the sense of Bochner integral, and the integrals of scalar valued continuous functions with respect to $X^{\prime}$-valued measures in the sense similar to that of Bochner discussed in the last section. 
Definition 3.1. Let $f \in C(\mathbb{T}, X)$ and $\mu \in M\left(\mathbb{T}, X^{\prime}\right)$. For every $n \in \mathbb{Z}$,

$$
\hat{f}(n):=\int_{\mathbb{T}} z^{-n} f(z) d z \quad \text { and } \quad \hat{\mu}(n):=\int_{\mathbb{T}} z^{-n} d \mu(z)
$$

are called the $n$ th-Fourier coefficient of $f$ and $\mu$, respectively.

For $f \in C(\mathbb{T}, X)$, let $\tau(f)$ denote the closed translation invariant subspace generated by $f$.

Proposition 3.2. $f \in C(\mathbb{T}, X)$ is mean-periodic if and only if $\tau(f) \neq C(\mathbb{T}, X)$.

Proof. Follows from the fact that the dual of $C(\mathbb{T}, X)$ is $M\left(\mathbb{T}, X^{\prime}\right)$.

LEMMA 3.3. For $f \in C(\mathbb{T}, X)$ and $\mu \in M\left(\mathbb{T}, X^{\prime}\right)$, the following hold:

(i) $f \star \mu$ is a uniformly continuous function on $\mathbb{T}$;

(ii) $(f \star \mu \hat{)}=\langle\hat{f}(n), \hat{\mu}(n)\rangle$.

PROOF. (i) Follows from the facts that $f$ is uniformly continuous, $\mu$ has finite variation and that $|(f \star \mu)(z)-(f \star \mu)(w)| \leq \int_{\mathbb{T}}\|f(z \bar{s})-f(w \bar{s})\| d V_{\mu}(s)$.

(ii) Since $\mathbb{T}$ is compact, $f$ is uniformly continuous on $\mathbb{T}$. Let $\epsilon_{k}>0$ be such that $\epsilon_{k} \rightarrow 0$ as $k \rightarrow \infty$. Since the metric on $\mathbb{T}$ is invariant under rotation, there exist finite Borel partitions $P_{k}$ of $\mathbb{T}=\sqcup B_{k i}$ such that if $z_{k i}, w_{k i} \in B_{k i}$, then $\| f\left(z_{k i} \bar{w}\right)-$ $f\left(w_{k i} \bar{w}\right) \|<\epsilon_{k}$ whenever $|w|=1$. Now

$$
\begin{aligned}
(f \star \mu)(n) & =\int_{\mathbb{T}}(f \star \mu)(z) z^{-n} d z=\int_{\mathbb{T}} \int_{\mathbb{T}} f(z \bar{w}) d \mu(w) z^{-n} d z \\
& =\int_{\mathbb{T}} \lim _{k \rightarrow \infty}\left(\sum_{j}\left\langle f\left(z \overline{w_{k j}}\right), \mu\left(B_{k j}\right)\right\rangle\right) z^{-n} d z .
\end{aligned}
$$

Since $f$ is continuous on $\mathbb{T}, f(\mathbb{T}) \subset B(0, r)=r B(0,1)$ for some $r>0$. We have

$$
\begin{aligned}
\left|\sum_{j}\left\langle f\left(z \overline{w_{k j}}\right), \mu\left(B_{k j}\right)\right\rangle\right| & \leq \sum_{j}\left|\left\langle f\left(z \overline{w_{k j}}\right), \mu\left(B_{k j}\right)\right\rangle\right| \\
& \leq \sum_{j} r V_{\mu}\left(B_{k j}\right) \leq r V_{\mu}(\mathbb{T}) \leq r C .
\end{aligned}
$$

Applying dominated convergence theorem in (14) for the functions

$$
z \mapsto \sum_{j}\left\langle f\left(z \overline{w_{k j}}\right), \mu\left(B_{k j}\right)\right\rangle z^{-n}
$$


we obtain

$$
\begin{aligned}
(f \star \mu)^{\hat{n}}(n) & =\lim _{k \rightarrow \infty} \int_{\mathbb{T}} \sum_{j}\left\langle f\left(z \overline{w_{k j}}\right), \mu\left(B_{k j}\right)\right\rangle z^{-n} d z \\
& =\lim _{k \rightarrow \infty} \sum_{j} \int_{\mathbb{T}}\left\langle f\left(z \overline{w_{k j}}\right), \mu\left(B_{k j}\right)\right\rangle z^{-n} d z \\
& =\lim _{k \rightarrow \infty} \sum_{j} \int_{\mathbb{T}}\left\langle z^{-n} f\left(z \overline{w_{k j}}\right), \mu\left(B_{k j}\right)\right\rangle d z .
\end{aligned}
$$

Now apply change of variable formula for the function $z \mapsto\left\langle z^{-n} f\left(z \overline{w_{k j}}\right), \mu\left(B_{k j}\right)\right\rangle$, to get

$$
\begin{aligned}
(f \star \mu)^{\hat{n}}(n) & =\lim _{k \rightarrow \infty} \sum_{j} \int_{\mathbb{T}}\left\langle\left(\frac{z}{\overline{w_{k j}}}\right)^{-n} f(z), \mu\left(B_{k j}\right)\right\rangle d z \\
& =\lim _{k \rightarrow \infty} \sum_{j}\left\langle\int_{\mathbb{T}}\left(\frac{z}{\overline{w_{k j}}}\right)^{-n} f(z) d z, \mu\left(B_{k j}\right)\right\rangle \\
& =\lim _{k \rightarrow \infty} \sum_{j}\left(\overline{w_{k j}}\right)^{-n}\left\langle\hat{f}(n), \mu\left(B_{k j}\right)\right\rangle \\
& =\left\langle\hat{f}(n), \lim _{k \rightarrow \infty} \sum_{j}\left(\overline{w_{k j}}\right)^{-n} \mu\left(B_{k j}\right)\right\rangle=\langle\hat{f}(n), \hat{\mu}(n)\rangle .
\end{aligned}
$$

COROllary 3.4. For $f \in C(\mathbb{T}, X)$ and $\mu \in M\left(\mathbb{T}, X^{\prime}\right), f \star \mu=0$ if and only if $\langle\hat{f}(n), \hat{\mu}(n)\rangle=0$ for all $n \in \mathbb{Z}$.

PROOF. Follows from Lemma 3.3 and the uniqueness of Fourier-Stieltjes coefficients of scalar valued functions on $\mathbb{T}$.

Proposition 3.5. Let $f \in C(\mathbb{T}, X)$. Then $\sigma(f)=\left\{\alpha z^{n} \hat{f}(n): \hat{f}(n) \neq 0\right.$ and $0 \neq$ $\alpha \in \mathbb{C}\}$.

PROOF. First we show that $\left\{\alpha z^{n} \hat{f}(n): \hat{f}(n) \neq 0\right\} \subseteq \sigma(f)$. Let $\mu \in M\left(\mathbb{T}, X^{\prime}\right)$ be such that $\mu(\tau(f))=0$. Then $f \star \mu=0$, since $\tau(f)$ is translation invariant. Hence by Corollary 3.4, $\langle\hat{f}(n), \hat{\mu}(n)\rangle=0$ for every $n$. Thus $\mu\left(\alpha z^{n} \hat{f}(n)\right)=\alpha\langle\hat{f}(n), \hat{\mu}(n)\rangle=0$, and by Corollary 3.4, $\alpha z^{n} \hat{f}(n) \in \tau(f)$. Hence $\alpha z^{n} \hat{f}(n) \in \sigma(f)$.

On the other hand, let $z^{m} x \in \sigma(f)$. To show that $x=\alpha \hat{f}(m)$ for some scalar $\alpha$. Let $x^{\prime} \in X^{\prime}$ be such that $x^{\prime}(\hat{f}(m))=0$. Let $d v(z)=z^{m} x^{\prime} d z$. Then

$$
\hat{\mu}(n)= \begin{cases}x^{\prime} & \text { if } n=m \\ 0 & \text { if } n \neq m .\end{cases}
$$


Thus by Corollary 3.4, $f \star v=0$. Therefore $z^{m} x \star v=0$ and hence $\langle x, \hat{v}(m)\rangle=0$, that is, $\left\langle x, x^{\prime}\right\rangle=0$. Thus for $x^{\prime} \in X^{\prime},\left\langle\hat{f}(m), x^{\prime}\right\rangle=0$ implies $\left\langle x, x^{\prime}\right\rangle=0$. Therefore $x=\alpha \hat{f}(m)$ for some $\alpha \in \mathbb{C}$. This completes the proof.

Proposition 3.6. Let $f \in C(\mathbb{T}, X)$. Then $\sigma(f)=\emptyset$ if and only if $f=0$.

PROOF. By Proposition 3.5, it suffices to show that $\hat{f}(n)=0$, for every $n \in \mathbb{Z}$ if and only if $f=0$. Using the uniqueness of Fourier coefficients for scalar valued functions we obtain, for every $n \in \mathbb{Z}$ and $x^{\prime} \in X^{\prime}$,

$$
\begin{aligned}
\hat{f}(n)=0 & \Leftrightarrow\left\langle x^{\prime}, \hat{f}(n)\right\rangle=0 \Leftrightarrow\left\langle x^{\prime}, \int_{\mathbb{T}} f(z) z^{-n} d z\right\rangle=0 \Leftrightarrow \int_{\mathbb{T}}\left\langle x^{\prime}, f(z)\right\rangle z^{-n} d z=0 \\
& \Leftrightarrow\left(x^{\prime} \circ f\right)^{\prime}(n)=0 \Leftrightarrow x^{\prime} \circ f=0 \Leftrightarrow f=0 .
\end{aligned}
$$

THEOREM 3.7. For a complex Banach space $X \neq \mathbb{C}$ the following hold:

(i) $M P(\mathbb{T}, X)=C(\mathbb{T}, X)$.

(ii) For every $0 \neq \mu \in M\left(\mathbb{T}, X^{\prime}\right),\{0\} \neq M P(\mu) \neq C(\mathbb{T}, X)$.

ProOF. (i) Let $f: \mathbb{T} \rightarrow X$ be a non zero continuous function. Then $\hat{f}\left(n_{0}\right) \neq 0$ for some $n_{0}$. Chose $x^{\prime} \in X^{\prime}$ such that $x^{\prime} \neq 0$ and $\left\langle x^{\prime}, \hat{f}\left(n_{0}\right)\right\rangle=0$. Define $\mu(E):=$ $\left(\int_{E} z^{n_{0}} d z\right) x^{\prime}$, for every $E \in \mathscr{B}_{\mathbb{T}}$. Then $\mu \in M\left(\mathbb{T}, X^{\prime}\right)$ and

$$
\hat{\mu}(n)= \begin{cases}x^{\prime} & \text { if } n=n_{0} \\ 0 & \text { if } n \neq n_{0}\end{cases}
$$

Thus $(f \star \mu \hat{)}(n)=\langle\hat{f}(n), \hat{\mu}(n)\rangle=0$, for every $n \in \mathbb{Z}$. Hence it follows from Corollary $3.4, f \star \mu=0$.

(ii) Let $0 \neq \mu \in M\left(\mathbb{T}, X^{\prime}\right)$. Then $\hat{\mu}\left(n_{0}\right) \neq 0$ for some $n_{0}$. Let $0 \neq x \in X$ be such that $\left\langle\hat{\mu}\left(n_{0}\right), x\right\rangle=0$, and $y \in X$ be such that $\left\langle\hat{\mu}\left(n_{0}\right), y\right\rangle \neq 0$. Define $f, g: \mathbb{T} \rightarrow X$, by $f(z)=z^{n_{0}} x$ and $g(z)=z^{n_{0}} y$. Then

$$
\hat{f}(n)=\left\{\begin{array}{ll}
x & \text { if } n=n_{0} ; \\
0 & \text { if } n \neq n_{0}
\end{array} \quad \text { and } \quad \hat{g}(n)= \begin{cases}y & \text { if } n=n_{0} \\
0 & \text { if } n \neq n_{0}\end{cases}\right.
$$

Therefore, $\langle\hat{f}(n), \hat{\mu}(n)\rangle=0$ for all $n \in \mathbb{Z}$ and $(g \star \mu)\left(n_{0}\right)=\left\langle\hat{g}\left(n_{0}\right), \hat{\mu}\left(n_{0}\right)\right\rangle \neq 0$. Thus $f$ is mean-periodic with respect to $\mu$ and $g$ is not mean-periodic with respect $\mu$.

REMARK. (1) Theorem 3.7 (i) is not true when $X=\mathbb{C}$. For instance, the function $f: \mathbb{T} \rightarrow \mathbb{C}$ defined by $f(z):=\sum_{-\infty}^{\infty} a_{n} z^{n}, z \in \mathbb{T}$, where $a_{n} \in \mathbb{C}, a_{n} \neq 0$ for every $n$ and $\sum_{-\infty}^{\infty}\left|a_{n}\right|<\infty$ is not mean-periodic. 
(2) Let $G$ be a locally compact abelian group and $X$ a complex Banach space. A function $f \in C(G, X)$ is said to be almost periodic if the set of all translates of $f$ is relatively compact in $C(G, X)$. Every $f \in C(\mathbb{T}, X)$ is almost periodic and if $X \neq \mathbb{C}$, then every $f \in C(\mathbb{T}, X)$ is mean-periodic. When $X=\mathbb{C}$, there are complex valued continuous functions on the circle group $\mathbb{T}$ which are not mean-periodic.

We have the following result for spectral analysis and spectral synthesis for $\mathbb{T}$.

THEOREM 3.8. The following hold:

(i) Let $x \in X, x \neq 0$, and $n_{0} \in \mathbb{Z}$. Then $\tau\left(z^{n_{0}} x\right)$, the closed translation invariant subspace generated by $z^{n_{0}} x$, does not contain any non-zero proper closed translation invariant subspace of $C(\mathbb{T}, X)$.

(ii) Every non-zero closed translation invariant subspace $V$ of $C(\mathbb{T}, X)$ contains an exponential, that is, spectral analysis holds in $C(\mathbb{T}, X)$.

(iii) The linear span of the exponentials in every closed translation invariant subspace $V$ of $C(\mathbb{T}, X)$ is dense in $V$, that is, spectral synthesis holds in $C(\mathbb{T}, X)$.

PROOF. (i) Let $V_{1}$ be a non-zero closed translation invariant subspace of $C(\mathbb{T}, X)$ such that $V_{1} \subseteq \tau\left(z^{n_{0}} x\right)$. Then for $f \in \tau\left(z^{n_{0}} x\right), \hat{f}\left(n_{0}\right)=c x$ for some $0 \neq c \in \mathbb{C}$ and $\hat{f}(n)=0$ if $n \neq n_{0}$. To show $V_{1}=\tau\left(z^{n_{0}} x\right)$, let $\mu \in M\left(\mathbb{T}, X^{\prime}\right)$ be such that $\mu\left(V_{1}\right)=\{0\}$. Then $\langle\hat{\mu}(n), x\rangle$ for every $n$. In particular $\left\langle\hat{\mu}\left(n_{0}\right), x\right\rangle$ and hence $\mu(V)=\{0\}$. Hence $V_{1}=\tau\left(z^{n_{0}} x\right)$.

(ii) Choose $n_{0} \in \mathbb{Z}$ and $f \in V$ such that $\hat{f}\left(n_{0}\right) \neq 0$. We will show that $z^{n_{0}} \hat{f}\left(n_{0}\right) \in V$. For, let $\mu \in M\left(\mathbb{T}, X^{\prime}\right)$ be such that $\mu(V)=\{0\}$. Since $V$ is translation invariant and $\mu(V)=\{0\}, f \star \mu=0$. This implies $\left\langle\hat{f}\left(n_{0}\right), \hat{\mu}\left(n_{0}\right)\right\rangle=0$. Thus $z^{n_{0}} \hat{f}\left(n_{0}\right) \star \mu=0$. Hence $z^{n_{0}} \hat{f}\left(n_{0}\right) \in V$.

(iii) Let $V$ be closed translation invariant subspace of $C(\mathbb{T}, X)$. Let $V_{0}$ be the closed linear span of $z^{n} \hat{f}(n), f \in V$. Then by (ii), $V_{0} \subseteq V$. Let $f \in V$. Let $\mu \in M\left(\mathbb{T}, X^{\prime}\right)$ such that $\mu\left(V_{0}\right)=0$. Then $\langle\hat{f}(n), \hat{\mu}(n)\rangle=0$, for every $n \in \mathbb{Z}$. Thus $f \star \mu=0$. Therefore, $\mu(f)=0$.

COROllary 3.9. For $f \in C(\mathbb{T}, X)$ and $\mu \in M\left(\mathbb{T}, X^{\prime}\right)$, the following are equivalent:

(i) $f \star \mu=0$.

(ii) $f$ is a limit of finite linear combinations of functions $z^{n} x$ which satisfy the equation $z^{n} x \star \mu=0$.

Proof. First observe that for a given $\mu, M P(\mu)=\{f \in C(\mathbb{T}, X): f \star \mu=$ $0\}$ is a closed translation invariant subspace of $C(\mathbb{T}, X)$. The result follows from Theorem 3.8 (iii). 


\section{Some results for general groups}

As mentioned earlier, problem of analysing mean-periodic functions, the problem of spectral analysis and spectral synthesis seems difficult to answer for general groups. However, it is not difficult to show that if $G$ is compact and $X=\mathbb{C}$ then every nontrivial closed translation invariant subspace $V$ of $C(K, \mathbb{C})$ includes exponentials and the linear span of exponentials in $V$ is dense in it. Hence every mean-periodic (scalar valued) function on a compact group is a limit of finite linear combination of exponentials.

For $G$ arbitrary locally compact abelian, and $X=\mathbb{C}$ we have the following: recall, $\Omega=\left\{\omega: G \rightarrow \mathbb{C}^{*}: \omega \in C(G)\right.$ and $\left.\omega\left(g_{1}+g_{2}\right)=\omega\left(g_{1}\right) \omega\left(g_{2}\right)\right\}$.

THEOREM 4.1. (i) Every $\omega \in \Omega$ is mean-periodic.

(ii) Let $G$ be an infinite locally compact $T_{1}$ abelian group. Then every exponential polynomial on $G$ is mean-periodic.

(iii) Let $M P(G)$ be the set of all mean-periodic functions on $G$. Then $M P(G)$ is dense in $C(G)$ if and only if $G$ is not finite.

PROOF. (i) Clearly, every translate $\omega_{g}$ of $\omega$ is a constant multiple of $\omega$, and hence every finite linear combination of translates of $\omega$ is a constant multiple of $\omega$. Therefore the closed translation invariant subspace $\tau(\omega)$ is a one dimensional subspace of $C(G)$. Thus $\tau(\omega) \neq C(G)$, if $G$ is non-trivial.

(ii) Let $f$ be an exponential polynomial on $G$,

$$
f(g):=\left(\sum_{\alpha} c_{\alpha} a_{1}(g)^{\alpha_{1}} a_{2}(g)^{\alpha_{2}} \cdots a_{m}(g)^{\alpha_{m}}\right) \omega(g),
$$

where $\alpha=\left(\alpha_{1}, \ldots, \alpha_{n}\right), \alpha_{i} \in \mathbb{N}, c_{\alpha}$ are complex constants and $a_{1}, \ldots, a_{m}$ are additive functions. Let $V=L S\left\{a_{1}(g)^{\beta_{1}} a_{2}(g)^{\beta_{2}} \cdots a_{m}(g)^{\beta_{m}} \omega(g): \beta_{j} \in \mathbb{Z}_{+}, \beta_{j} \leq \alpha_{j}\right.$ for $1 \leq$ $j \leq m\}$. It is easy to see that $f \in V$ and $V$ is a finite dimensional translation invariant subspace of $C(G)$. Since $V$ is finite dimensional, it is closed and it follows that $\tau(f) \subseteq V$. But $C(G)$ is infinite dimensional as $G$ is not finite. Hence $\tau(f) \neq C(G)$.

(iii) Suppose that $G$ is finite, $G=\left\{g_{1}, g_{2}, \ldots, g_{n}\right\}$. Let $f \in C(G)$ and $\mu \in M_{c}(G)$. Let $\mu\left(g_{i}\right)=c_{i}$. Then $f \star \mu=0$ for a non-trivial $\mu$ if and only if

$$
\left|\begin{array}{cccc}
f\left(g_{1}-g_{1}\right) & f\left(g_{1}-g_{2}\right) & \cdots & f\left(g_{1}-g_{n}\right) \\
f\left(g_{2}-g_{1}\right) & f\left(g_{2}-g_{2}\right) & \cdots & f\left(g_{2}-g_{n}\right) \\
\vdots & \vdots & & \vdots \\
f\left(g_{n}-g_{1}\right) & f\left(g_{n}-g_{2}\right) & \cdots & f\left(g_{n}-g_{n}\right)
\end{array}\right|=0 .
$$

The columns of the above matrix are permutations of $\left[f\left(g_{1}\right), f\left(g_{2}\right), \ldots, f\left(g_{n}\right)\right]$. Thus $f$ is mean-periodic if and only if $\left(f\left(g_{1}\right), f\left(g_{2}\right), \ldots, f\left(g_{n}\right)\right)$ is a root of some 
fixed polynomial $P$ in the variables $z_{1}, z_{2}, \ldots, z_{n}$. The roots of this polynomial $P$ form a closed set $Z(P)$ in $\mathbb{C}^{n}$ of $2 n$-dimensional Lebesgue measure zero. Therefore $Z(P)$ is not dense in $\mathbb{C}^{n}$. But $M P(G)=Z(P)$. Hence $M P(G)$ is not dense in $C(G)$.

Conversely, suppose that $G$ is not finite. Let $E P(G)$ be the set of all exponential polynomials in $C(G)$. By (ii), $E P(G) \subseteq M P(G)$, that is, $\Gamma \subseteq \Omega \subseteq E P(G) \subseteq$ $M P(G)$. Moreover $\Omega$ separates points of $G$. Since the pointwise product of finite number of exponentials is again an exponential, it is easy to see that product of two exponential polynomials $f$ and $g$ is a finite sum of exponential polynomials and hence $\tau(f g)$ is finite dimensional. Therefore the algebra $A(E P(G))$, generated by $E P(G)$, is contained in $M P(G)$, that is, $A(E P(G)) \subseteq M P(G)$. Hence by Stone Weierstrass theorem ([9]) $A(E P(G))$ is dense in $C(G)$. Since $A(E P(G)) \subseteq M P(G), M P(G)$ is dense in $C(G)$.

COROLLARY 4.2. If $G$ is a finite $T_{1}$ topological abelian group, then $\{0\} \neq M P(G)$ $\neq C(G)$.

LEMMA 4.3. Let $G$ be a locally compact abelian group having no nontrivial compact subgroups. Let $\hat{G}$ be the dual group of $G$. Then for $\mu \in M_{c}(G)$, $\lambda_{\Gamma}(\{\gamma \in \Gamma: \hat{\mu}(\gamma)=0\})=0$.

PROOF. Refer [6].

THEOREM 4.4. If $G$ does not have compact elements, then $\{0\} \neq M P(G) \neq C(G)$.

ProOF. Let $f \in C(G)$ be compactly supported. By Lemma 4.3, $f$ is not meanperiodic. Thus $M P(G) \neq C(G)$.

As we have pointed earlier, the problem of spectral synthesis does not hold for every closed translation invariant subspace $V$ of $C\left(\mathbb{R}^{2}, \mathbb{C}\right)$. However, with some conditions on $V$ this is true. First we prove the following lemma.

LEMMA 4.5. The following hold:

(i) Let $\lambda_{1}, \lambda_{2}, \ldots, \lambda_{n}$ be distinct complex numbers and $m_{1}, m_{2}, \ldots, m_{n} \in \mathbb{N}$. Then the set $\left\{e^{i \lambda_{j} t}, t e^{i \lambda_{j} t}, \ldots, t^{m_{j}} e^{i \lambda_{j} t}: 1 \leq j \leq n\right\} \subseteq C(\mathbb{R})$ is linearly independent over $\mathbb{C}$.

(ii) Let $\lambda_{1}, \lambda_{2}, \ldots, \lambda_{n} ; \eta_{1}, \eta_{2}, \ldots, \eta_{n}$ be complex numbers and for $1 \leq j, k, l \leq$ $n, \alpha_{l j}, \beta_{k r}$ be non-negative integers. Then $\left\{t_{1}^{\alpha_{l j}} t_{2}^{\beta_{l j}} e^{l\left(\lambda_{l} t_{1}+\eta_{l} t_{2}\right)}: 1 \leq l, j \leq n\right\}$ is a linearly independent subset of $C\left(\mathbb{R}^{2}\right)$ over $\mathbb{C}$ if $\left(\lambda_{j}, \eta_{j}\right) \neq\left(\lambda_{k}, \eta_{k}\right)$ or $\left(\alpha_{l j}, \beta_{l j}\right) \neq\left(\alpha_{l k}, \beta_{l k}\right)$.

PROOF. (i) Without loss of generality, we may assume that

$$
\operatorname{Im}\left(\lambda_{n}\right)=\max _{1 \leq j \leq n} \operatorname{Im}\left(\lambda_{j}\right),
$$


where $\operatorname{Im}$ denotes the imaginary part of a complex number. $\operatorname{Then} \operatorname{Im}\left(\lambda_{n}\right)-\operatorname{Im}\left(\lambda_{j}\right)>0$ for $1 \leq j \leq n-1$. Now for $a_{i j} \in \mathbb{C}$,

$$
\begin{gathered}
\sum_{j=1}^{n}\left(a_{0 j} e^{i \lambda_{j} t}+a_{1 j} t e^{\imath \lambda_{j} t}+\cdots+a_{m_{j} j} t^{m_{j}} e^{i \lambda_{j} t}\right)=0 \Longrightarrow \\
\sum_{j=1}^{n-1}\left(a_{0 j} e^{\iota\left(\lambda_{j}-\lambda_{n}\right) t}+a_{1 j} t e^{\iota\left(\lambda_{j}-\lambda_{n}\right) t}+\cdots+a_{m_{j} j} t^{m_{j}} e^{\iota\left(\lambda_{j}-\lambda_{n}\right) t}\right)+p_{n}(t)=0,
\end{gathered}
$$

where $p_{n}(t)=a_{n 0}+a_{n 1} t+\cdots+a_{n m_{n}} t^{m_{n}}=a_{n m_{n}}\left(t-\beta_{1}\right)\left(t-\beta_{2}\right) \cdots\left(t-\beta_{n_{m}}\right)$, for some $\beta_{1}, \beta_{2}, \ldots, \beta_{n_{m}} \in \mathbb{C}$. Now as $t \rightarrow-\infty, t^{k} e^{\ell\left(\lambda_{j}-\lambda_{n}\right)} \rightarrow 0$ for every $j \neq n$. This implies $a_{n m_{n}}=0$, since as $t \rightarrow-\infty, p_{n}(t) \nrightarrow \rightarrow 0$ if $a_{n m_{n}} \neq 0$. Similarly by repeating the same argument one can easily show that $a_{i j}=0$ for all $i, j$.

(ii) Case (i): $\lambda_{1}, \lambda_{2}, \ldots, \lambda_{n}$ are distinct. For $a_{l j} \in \mathbb{C}$,

$$
\sum_{l=1}^{n} \sum_{j=1}^{n}\left(a_{l j} t_{1}^{\alpha_{l j}} t_{2}^{\beta_{l j}} e^{l\left(\lambda_{l} t_{1}+\eta_{l} t_{2}\right)}\right)=0 \quad \Longrightarrow \quad \sum_{l=1}^{n} \sum_{j=1}^{n}\left(\left(a_{l j} t_{2}^{\beta_{l j}} e^{l \eta_{l} t_{2}}\right) t_{1}^{\alpha_{l j}} e^{l \lambda_{l} t_{1}}\right)=0 .
$$

By (i), this implies $a_{i j}=0$ for all $i, j$.

Case (ii): $\lambda_{i}=\lambda_{j}$ for some $i$ and $j$. In this case rearrange the terms of the above expression by collecting the distinct exponential monomials in $t_{1}$. By the hypothesis, the coefficients of the exponential monomial in $t_{1}$ are finite linear combination of exponential monomials in $t_{2}$. By applying (i) twice, namely, first $t_{1}$ variable and then $t_{2}$ variable we get $a_{i j}=0$ for all $i, j$.

THEOREM 4.6. Let $V$ be a closed translation invariant subspace of $C\left(\mathbb{R}^{2}\right)$ satisfying any one of the following conditions:

(i) $V$ is finite dimensional.

(ii) $V$ is rotation invariant.

(iii) $V=\tau_{\mu}:=\left\{f \in C\left(\mathbb{R}^{2}\right): f \star \mu=0\right\}$ for some $\mu \in M_{c}\left(\mathbb{R}^{2}\right)$.

Then $V$ contains an exponential.

Proof. Case (i): $V$ is finite dimensional. Let $f \in V$ and $f \neq 0$. By Theorem 1.9, $f$ is of the form $f=\sum_{j=1}^{m} p_{j}\left(t_{1}, t_{2}\right) e^{\iota\left(\lambda_{j} t_{1}+\eta_{j} t_{2}\right)}$, where $p_{j}$ is a non-zero polynomial in $t_{1}, t_{2}$ and $\left(\lambda_{j}, \eta_{j}\right) \neq\left(\lambda_{k}, \eta_{k}\right)$ for $j \neq k$. Let $\mu \in M_{c}\left(\mathbb{R}^{2}\right)$ be such that $\mu(V)=\{0\}$. We show that $\mu\left(e^{\iota\left(\lambda_{j} t_{1}+\eta_{j} t_{2}\right)}\right)=0$. Since $V$ is translation invariant, $f \star \mu=0$. Write $f$ as a linear combination of elements in $\left\{t_{1}^{\alpha_{l j}} t_{2}^{\beta_{l j}} e^{l\left(\lambda_{l} t_{1}+\eta_{t} t_{2}\right)}: 1 \leq l, j \leq n\right\}$. Let $c_{k_{1}} t_{1}^{\alpha_{0}} t_{2}^{\beta_{k_{1}}} e^{\iota\left(\lambda_{j} t_{1}+\eta_{j} t_{2}\right)}, c_{k_{2}} t_{1}^{\alpha_{0}} t_{2}^{\beta_{k_{2}}} e^{l\left(\lambda_{j} t_{1}+\eta_{j} t_{2}\right)}, \ldots, c_{k_{m}} t_{1}^{\alpha_{0}} t_{2}^{\beta_{k_{m}}} e^{\iota\left(\lambda_{j} t_{1}+\eta_{j} t_{2}\right)}$ be the terms containing $e^{\ell\left(\lambda_{j} t_{1}+\eta_{j} t_{2}\right)}$ and the largest degree term of $t_{1}$, namely $t_{1}^{\alpha_{0}}$, where $c_{k_{1}}, c_{k_{2}}, \ldots, c_{k_{m}}$ are non-zero scalars. Also, $f \star \mu$ has the same representation and the terms containing $t^{\alpha_{0}} e^{l\left(\lambda_{j} t_{1}+\eta_{j} t_{2}\right)}$ are $c_{k_{1}} \hat{\mu}\left(\lambda_{j}, \eta_{j}\right) t_{1}^{\alpha_{0}} t_{2}^{\beta_{k_{1}}} e^{l\left(\lambda_{j} t_{1}+\eta_{j} t_{2}\right)}, c_{k_{2}} \hat{\mu}\left(\lambda_{j}, \eta_{j}\right) t_{1}^{\alpha_{0}} t_{2}^{\beta_{k_{2}}} e^{l\left(\lambda_{j} t_{1}+\eta_{j} t_{2}\right)}, \ldots$, 
$c_{k_{m}} \hat{\mu}\left(\lambda_{j}, \eta_{j}\right) t_{1}^{\alpha_{0}} t_{2}^{\beta_{k_{m}}} e^{\iota\left(\lambda_{j} t_{1}+\eta_{j} t_{2}\right)}$. Since $f \star \mu=0$ and $c_{k_{j}} \neq 0, \hat{\mu}\left(\lambda_{j}, \eta_{j}\right)=0$, by Lemma 4.5. Therefore $\mu\left(e^{\iota\left(\lambda_{j} t_{1}+\eta_{j} t_{2}\right)}\right)=0$. Thus $e^{\iota\left(\lambda_{j} t_{1}+\eta_{j} t_{2}\right)} \in V$.

Cases (ii) and (iii): $V$ is rotation invariant, or $V=\tau_{\mu}$. By Theorem 1.7 and Theorem 1.8, $V$ contains an exponential polynomial. It follows easily from the proof of (i) that $V$ contains an exponential.

\section{References}

[1] P. M. Anselone and J. Korevaar, 'Translation invariant subspaces of finite dimension', Proc. Amer. Math. Soc. 15 (1964), 747-752.

[2] S. C. Bagchi and A. Sitaram, 'Spherical mean-periodic functions on semi simple Lie groups', Pacific J. Math. 84 (1979), 241-250.

[3] C. A. Berenstein and B. A. Taylor, 'Mean-periodic functions', Internat. J. Math. Math. Sci. 3 (1980), 199-235.

[4] L. Brown, B. M. Schreiber and B. A. Taylor, 'Spectral synthesis and the Pompeiu problem', Ann. Inst. Fourier (Grenoble) 23 (1973), 125-154.

[5] J. Delsarte, 'Les fonctions moyenne-periodiques', J. Math. Pures Appl. 14 (1935), 403-453.

[6] P. Devaraj and I. K. Rana, 'Relation between Pompeiu groups and mean-periodic groups', preprint, 2000.

[7] D. G. Dickson, 'Analytic mean-periodic functions', Trans. Amer. Math. Soc. 14 (1972), 361-374.

[8] J. Diestel and J. J. Uhl Jr., Vector measures, Math. Surveys Monographs 15 (Amer. Math. Soc., Providence RI, 1977).

[9] J. Dugunji, Topology (Prentice-Hall, New Delhi, 1975).

[10] L. Ehrenpreis, 'Appendix to the paper 'Mean-periodic functions I", Amer. J. Math. 77 (1955), $731-733$.

[11] - 'Mean-periodic functions, Part I. Varieties whose annihilator ideals are principal', Amer. J. Math. 77 (1955), 293-328.

[12] R. J. Elliott, 'Some results in spectral synthesis', Proc. Camb. Phil. Soc. 61 (1965), 395-424.

[13] — 'Two notes on spectral synthesis for discrete abelian groups', Proc. Camb. Phil. Soc. 61 (1965), 617-620.

[14] M. Engert, 'Finite dimensional translation invariant subspaces', Pacific J. Math. 32 (1970), 333343.

[15] J. E. Gilbert, 'Spectral synthesis problems for invariant subspaces on groups II', in: Proc. Int. Sym. on Function Algebras at Tulane Univ. (1965) pp. 257-264.

[16] - 'Spectral synthesis problems for invariant subspaces on groups I', Amer. J. Math. 88(1966), 626-635.

[17] D. L. Gurevich, 'Counter examples to a problem of L. Schwartz', Funct. Anal. Appl. 9 (1975), 116-120.

[18] E. Hille and R. S. Phillips, Functional analysis and semi-groups, Amer. Math. Soc. Colloq. Publ. 21 (Amer. Math. Soc., Providence, RI, 1957).

[19] J. P. Kahane, Lectures on mean-periodic functions (Tata Institute, 1957).

[20] P. Koosis, 'On functions which are mean-periodic on a half-line', Comm. Pure Appl. Math. 10 (1957), 133-149.

[21] G. E. Ladas and V. Lakshmikantham, Differential equations in abstract spaces (Academic Press, New York, 1972). 
[22] P. G. Laird, 'Some properties of mean-periodic functions', J. Austral. Math. Soc. 14 (1972), 424-432.

[23] _ 'Functional differential equations and continuous mean-periodic functions', J. Math. Anal. Appl. 47 (1974), 406-423.

[24] — 'Entire mean-periodic functions', Canad. J. Math. 17 (1975), 805-818.

[25] - 'On characterisations of exponential polynomials', Pacific J. Math. 80 (1979), 503-507.

[26] M. Lefranc, 'L' analysis harmonique dans $\mathbb{Z}^{n}$ ', C. R. Acad. Sci. Paris 246 (1958), 1951-1953.

[27] B. M. Levitan and V. V. Zhikov, Almost periodic functions and differential equations (Cambridge University Press, Cambridge, 1982).

[28] B. Malgrange, 'Sur quelques propriètès des equations des convolution', C. R. Acad. Sci. Paris 238 (1954), 2219-2221.

[29] A. Meril, 'Analytic functions with unbounded carriers and mean-periodic functions', Trans. Amer. Math. Soc. 278 (1983), 115-136.

[30] Y. Meyer, 'Harmonic analysis of mean-periodic functions', in: Studies in harmonic analysis, MAA Stud. Math. 13 (Math. Assoc. Amer., Washington D.C., 1976) pp. 151-160.

[31] E. Novak and I. K. Rana, 'On the unsmoothing of functions on the real line', Proc. Nede. Acad. Sci. Ser. A 89 (1986), 201-207.

[32] I. K. Rana, 'Unsmoothing over balls via plane wave decomposition', Rend. Circ. Mat. Palermo (2) 34 (1990), 217-234.

[33] I. K. Rana and N. Gowri, 'Integrable mean-periodic functions on locally compact abelian groups', Proc. Amer. Math. Soc. 117 (1993), 405-410.

[34] J. Schmets, Spaces of vector valued continuous functions, Lecture Notes in Math. 1003 (Springer, 1983).

[35] L. Schwartz, 'Theorie generale des fonctions moyenne-periodiques', Ann. of Math. (2) 48 (1947), 857-929.

[36] H. S. Shapiro, 'The expansions of mean-periodic functions in series of exponentials', Comm. Pure Appl. Math. 11 (1958), 1-21.

[37] L. Székelyhidi, 'The Fourier transform of mean-periodic functions', Utilitas Math. 29 (1986), 43-48.

[38] - Convolution type functional equations on topological abelian groups (World Scientific Publishing, Singapore, 1991).

[39] Y. Weit, 'On Schwartz theorem for the motion group', Ann. Inst. Fourier (Grenoble) 30 (1980), 91-107.

Department of Mathematics

Indian Institute of Technology

Powai

Mumbai-76

PIN-400076

India

e-mail: devaraj@math.iitb.ac.in,ikr@math.iitb.ac.in 